
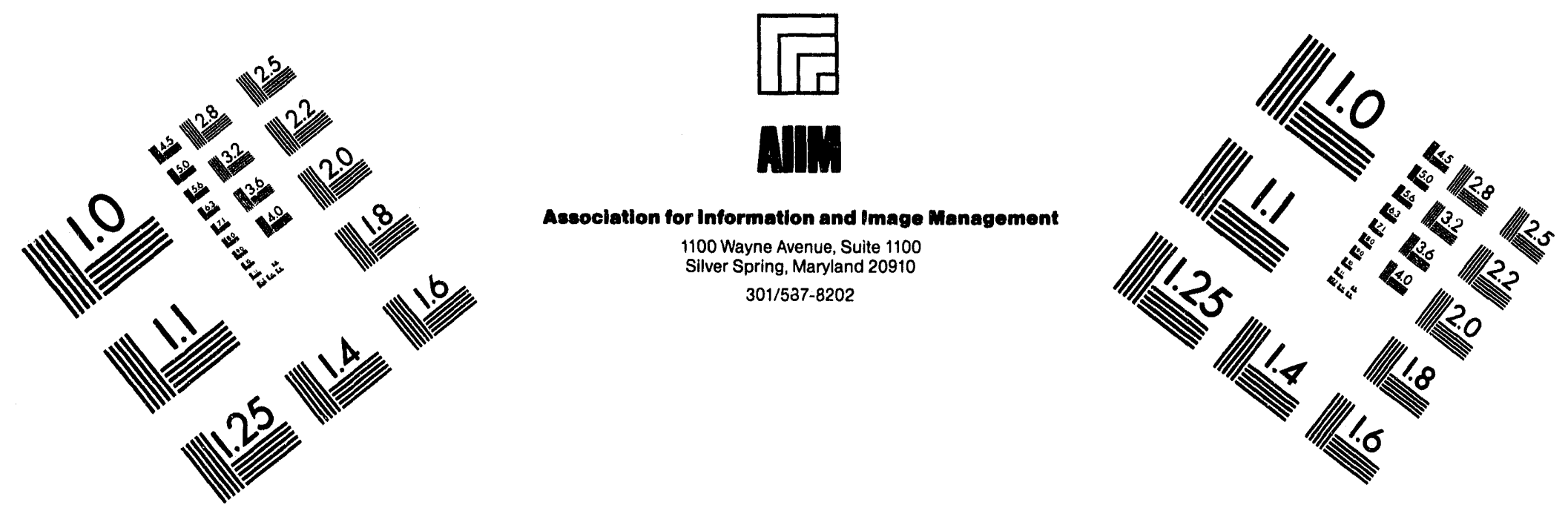

\title{
Centimeter
}

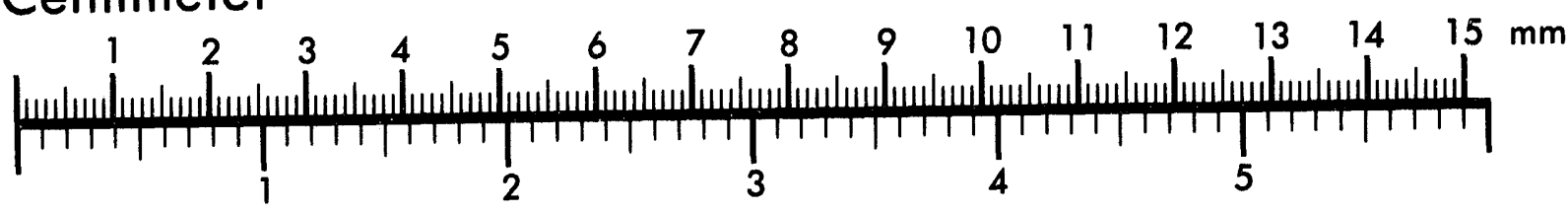
Inches
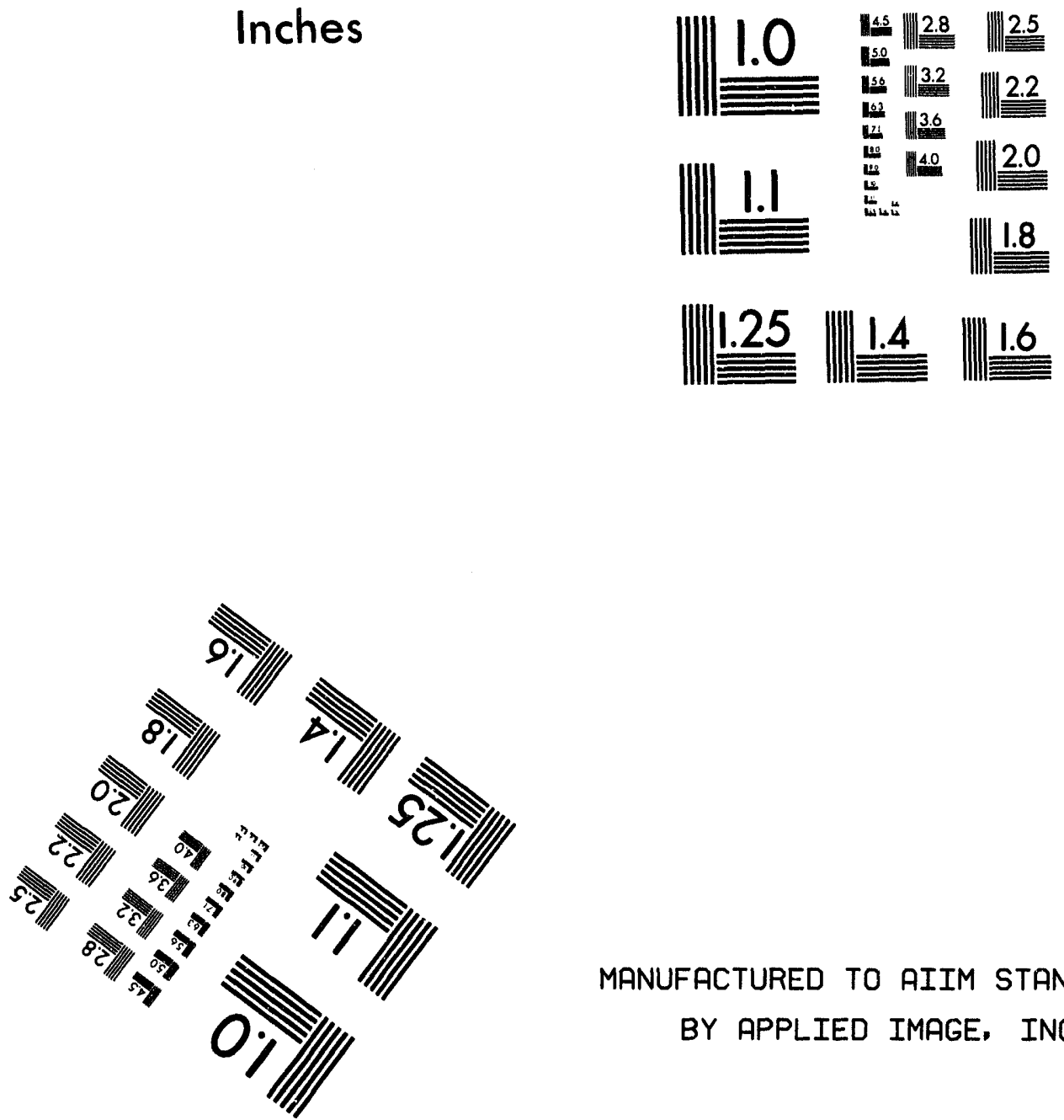

MANUFACTURED TO AIIM STANDARDS

BY APPLIED IMAGE, INC.

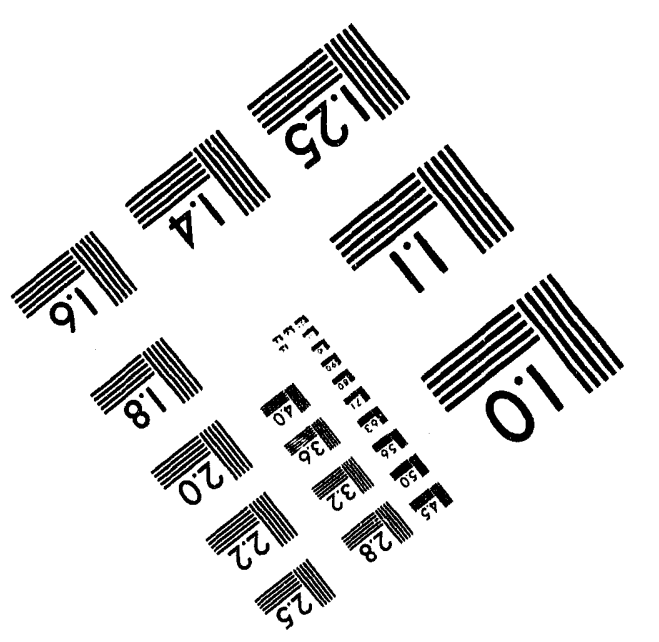




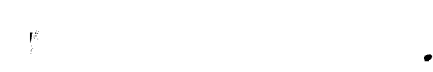

$m$
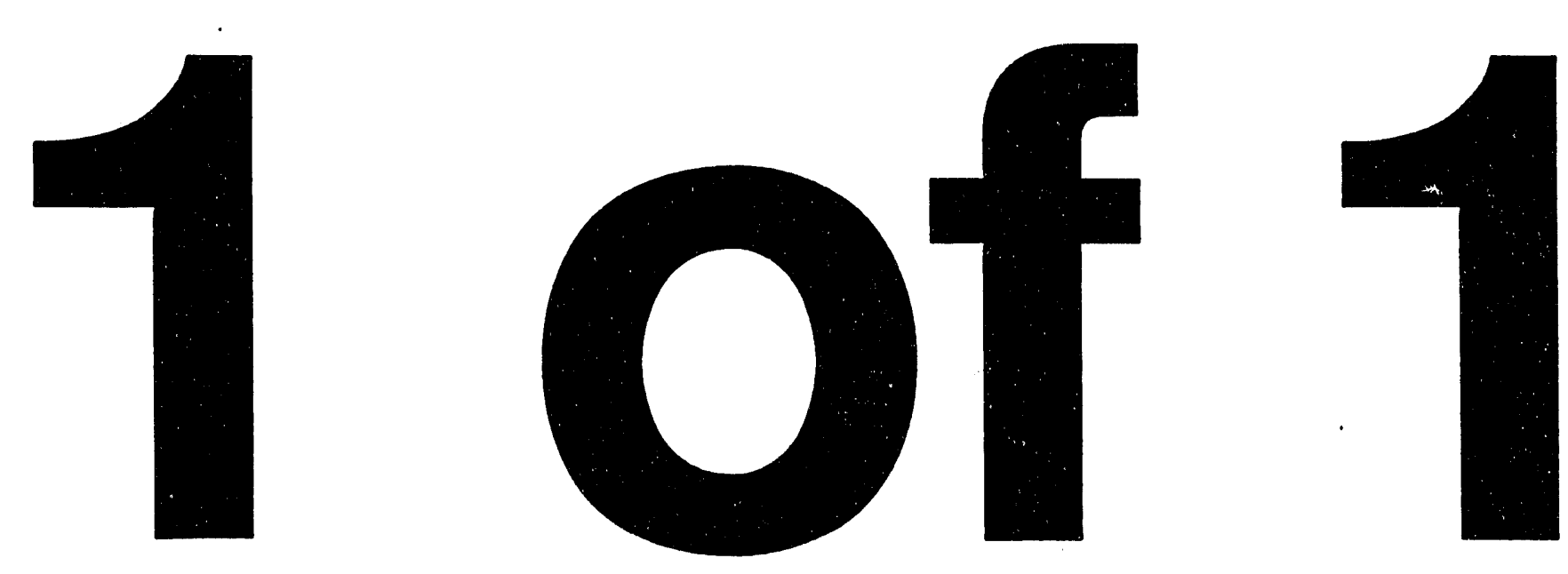
NUREG/CR-6176

ANL-94/1

\section{Review of Environmental Effects on Fatigue Crack Growth of Austenitic Stainless Steels}

Manuscript Completed: December 1993

Date Published: May 1994

Prepared by

W. J. Shack, T. F. Kassner

Argonne National Laboratory

9700 South Cass Avenue

Argonne, IL 60439

Prepared for

Office of Nuclear Reactor Regulation

U.S. Nuclear Regulatory Commission

Washington, DC 20555-0001

NRC FIN L2424 


\title{
Review of Environmental Effects on Fatlgue Crack Growth of Austenitic Stainless Steels
}

by

W. J. Shack and T. F. Kassner

\begin{abstract}
Fatigue and environmentally assisted cracking of piping, pressure vessel cladding, and core components in light water reactors are potential concerns to the nuclear industry and regulatory agencies. The degradation processes include intergranular stress corrosion cracking of austenitic stainless steel (SS) piping in boiling water reactors (BWRs), and propagation of fatigue or stress corrosion cracks (which inttiate in sensitized SS cladding) into low-alloy ferritic steels in BWR pressure vessels. Crack growth data for wrought and cast austenitic SSs in simulated BWR water, developed at Argonne National Laboratory under U.S. Nuclear Regulatory Commission sponsorship over the past 10 years, have been complled into a data base along with similar data obtained from the open literature. The data were analyzed to develop corrosion-fatigue curves for austenitic SSs in aqueous environments corresponding to normal BWR water chemistrles, for BWRs that add hydrogen to the feedwater, and for pressurized water reactor primary-system-coolant chemistry. The corrosion-fatigue data and curves in water were compared with the air line in Section XI of the ASME Code.
\end{abstract}




\section{Contents}

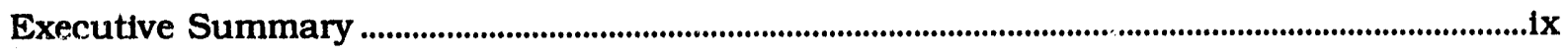

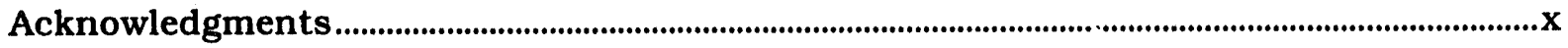

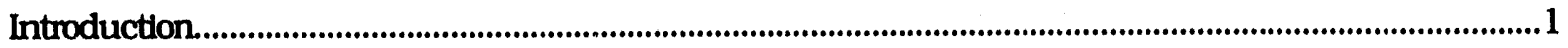

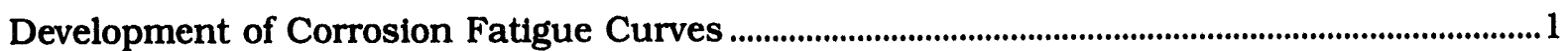

Appendix A: ANL Crack Growth Data Base for Wrought and Cast Austenitic

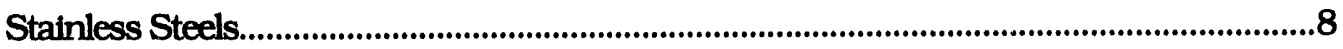

Appendix B: Effects of Water Chemistry on CGRs of Austenitic Stainless Steels.................17

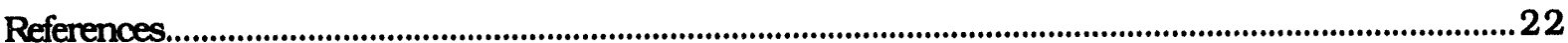




\section{Figures}

1. Corrosion fatigue data for wrought SSs in water containing $200 \mathrm{ppb}$ DO at $288^{\circ} \mathrm{C}$ compared with models.

2. Corrosion fatigue data for wrought SSs in water containing $8 \mathrm{ppm}$ DO at $288^{\circ} \mathrm{C}$ compared with models

3. Corrosion fatigue data for sensitized and nonsensitized SSs in water.. containing $200 \mathrm{ppb} \mathrm{DO}$ at $288^{\circ} \mathrm{C}$

4. Corrosion fatigue data for cast SSs in water containing $200 \mathrm{ppb}$ DO at $288^{\circ} \mathrm{C}$ compared with model

5. Corrosion fatigue data for cast SSs in water containing $8 \mathrm{ppm} \mathrm{DO}$ at $288^{\circ} \mathrm{C}$ compared with model.

6. Corrosion fatigue data for wrought SSs in water containing low DO at $288^{\circ} \mathrm{C}$, superposition models, and a model of Ford et al.

7. Corrosion fatigue data for wrought SSs in simulated PWR water and curves from models based on data obtained in simulated BWR water 5

8. Current model and model proposed by Gilman et al. in water containing $200 \mathrm{ppb}$ and $8 \mathrm{ppm}$ DO

9. Regime of high and low CGRs for moderately sensitized Type 304 SS specimen at $289^{\circ} \mathrm{C}$ as a function of ratio of chromate and sulfate concentrations in feedwater containing $\approx 200 \mathrm{ppb}$ DO.

10. ANL corrosion fatigue data at $R \geq 0.95$ for sensitized Type 304 SS in $289^{\circ} \mathrm{C}$ water containing $200 \mathrm{ppb}$ DO at several conductivity levels

11. ANL corrosion fatigue data at $R \leq 0.92$ for sensitized Type 304 SS in $289^{\circ} \mathrm{C}$ water containing $200 \mathrm{ppb}$ DO at several conductivity levels

12. Corrosion fatigue data in water containing $\mathbf{2 0 0} \mathrm{ppb}$ DO and chromate and sulfate at low concentrations where the $\left[\mathrm{CrO}_{4}^{2-}\right] /\left[\mathrm{SO}_{4}^{2-}\right]=>3$. 


\section{Tables}

1. Summary of equations for corrosion fatigue in BWR and PWR

environments

2. Corrosion fatigue curves in BWR and PWR environments in da/dN form...................7

3. Summary of crack growth results for Type 316NG and sensitized and solution-annealed Type 304 SS specimens in oxygenated $(\approx 200 \mathrm{ppb})$ water at $289^{\circ} \mathrm{C}$, in which load ratio and stress intensity were varled......

4. Summary of crack growth results for solution-annealed and sensitized Type 304 SS specimens in oxygenated $(\approx 5-8 \mathrm{ppm})$ water at $289^{\circ} \mathrm{C}$, in which load ratio, frequency, and stress intensity were varied.

5. Summary of crack growth results for Type 316NG and sensitized and solution annealed Type 304 SS specimens in oxygenated $(\approx 200 \mathrm{ppb})$ water with ionic impurities at $289^{\circ} \mathrm{C}$, in which load ratio and stress intensity were varied.

6. Summary of crack growth results for Type 347 SS specimens in oxygenated water at $289^{\circ} \mathrm{C}$, in which load ratio and stress intensity were varied.

7. Summary of crack growth results for cast SS specimens in water at $289^{\circ} \mathrm{C}$, in which load ratio and stress intensity were varied 


\section{Executlve Summary}

Fatigue and environmentally assisted cracking of piping, pressure vessel cladding, and core components in light water reactors are potential concerns to the nuclear industry and regulatory agencies. The degradation processes include intergranular stress corrosion cracking of austenitic stainless steel (SS) piping in boiling water reactors (BWRs), and propagation of fatigue or stress corrosion cracks (which initiate in sensitized SS cladding) into low-alloy ferritic steels in BWR pressure vessels. Crack growth data for wrought and cast austenitic SSs in simulated BWR water, developed at Argonne National Laboratory under U.S. Nuclear Regulatory Commission sponsorship over the past 10 years, have been compiled into a data base along with similar data obtained from the open literature. The data were analyzed to develop corrosion-fatigue curves for austenitic SSs in aqueous environments corresponding to normal BWR water chemistries (NWCs), for BWRs that add hydrogen to the feedwater, and for pressurized water reactor (PWR) primary-system-coolant chemistry. The corrosion-fatigue data and curves in water were compared with the air line in Section XI of the ASME Code.

Under most loading conditions, the contribution from stress corrosion cracking is negligible for PWR primary-water-chemistry conditions and for BWRs that employ hydrogen-water chemistry (HWC). The available laboratory data show that at crack growth rates (CGRs) of $<10^{-10} \mathrm{~m} \cdot \mathrm{s}^{-1}\left(0.25 \mathrm{in} \cdot \mathrm{yr}^{-1}\right)$, the rates in BWR NWCs exceed the air line in the ASME Code by a factor of $\approx 20-30$. Relatively few data are avallable in PWR environments at rates of $<10^{-9} \mathrm{~m} \cdot \mathrm{s}^{-1}$. At high CGRs the observed enhancement in both PWR and BWR environments is relatively small, and the magnitude of the enhancement under the same loading conditions is comparable in the two environments. Until further data are obtained for PWR water at low CGRs, we recommended that the environmental enhancement in BWR environments with HWC should be considered for PWR environments. 


\section{Acknowledgments}

This work was supported by the License Renewal Project Directorate, Office of Nuclear Reactor Regulation, U.S. Nuclear Regulatory Commission (NRC), under FIN L2424; Program Manager: D. Jackson. The crack growth data base on wrought and cast stainless steels in simulated BWR water and the correlations for estimating corrosion-fatigue properties of the steels have been developed over a period of 10 years as part of a program entitled "Environmentally Assisted Cracking and Fatigue in LWR Systems," sponsored by the Office of Nuclear Regulatory Research, U.S. NRC, under FIN A2212; Program Manager: Joe Muscara. The authors are thankful to Sam Lee for helpful discussions. 


\section{Introduction}

In-service inspection of piping and pressure vessels in light water reactors (LWRs) occasionally reveals the presence of planar flaws (l.e., weld defects or stress corrosion cracks) that must be addressed to determine whether a plant can be operated safely for a given time period before the flaw is repaired or the component is replaced. Reactor piping systems are designed in accordance with Section III of the ASME Code to ensure that they have adequate resistance to initiation of fatigue cracks. However, once a flaw is detected and its size and depth are determined, the extent to which the flaw will grow during continued service can be determined by procedures outlined in Section XI of the ASME Code. At present. Section XI of the Code does not explicitly account for effects of reactor coolant environments on crack growth rates (CGRs) of austenitic stainless steels (SSs). This report summarkes avallable data on growth of fatigue cracks and provides correlations for assessing fatigue-crack propagation in austenitic SS piping in bolling-water-reactor (BWR) and pressurized-water-reactor (PWR) environments.

\section{Development of Corrosion Fatigue Curves}

Most of the avallable data on corrosion fatigue of austenitic SSs in aqueous environments have been developed to support LWR technology in the U.S. and abroad. Because Section XI of the ASME Code currently provides only an in-air design curve, corrosion-fatigue data for wrought and cast SSs in simulated BWR environments, obtained at Argonne National Laboratory (ANL) (Appendix A) and from the literature, have been analyzed to develop corrosion-fatigue curves for SSs in aqueous environments. 1 The approach is basically an update of the work of Gilman et al..2 incorporating additional data that are now available.

The CGR, $\dot{a}_{\text {super, }}$ in an aqueous environment, is written as a superposition of a term representing the contribution of stress corrosion cracking (SCC) under constant load, àscc: a corrosion-fatigue term, a env, representing the additional CGR under cyclic loading due to the environment; and a mechanical fatigue term $\dot{a}_{\text {air }}$. representing the fatigue-crack growth in air,

$$
\dot{\mathrm{a}}_{\text {super }}=\dot{\mathrm{a}}_{\mathrm{SCC}}+\dot{\mathrm{a}}_{\mathrm{env}}+\dot{\mathrm{a}}_{\mathrm{air}}
$$

For the SCC term, the correlation given in U.S. Nuclear Regulatory Commission (NRC) Report NUREG-0313, Rev. 2, January 1988, is used for water chemistries with 8 ppm dissolved oxygen (DO): for water chemistries with $200 \mathrm{ppb}$ oxygen, the CGR is taken as onethird that given in NUREG-0313:

$$
\begin{array}{lll}
\dot{a}_{\mathrm{SCC}}=2.1 \times 10^{-13} \mathrm{~K}^{2.161} & \left(\mathrm{~m} \cdot \mathrm{s}^{-1}\right) & 8 \mathrm{ppm} \mathrm{DO} \\
\dot{\mathrm{a}}_{\mathrm{SCC}}=7.0 \times 10^{-14} \mathrm{~K}^{2.161} & \left(\mathrm{~m} \cdot \mathrm{s}^{-1}\right) & 200 \mathrm{ppb} \mathrm{DO},
\end{array}
$$

where $\mathrm{K}$ is the stress intensity factor in $\mathrm{MPa} \cdot \mathrm{m}^{1 / 2}$. The contribution from $\mathrm{SCC}$ is assumed to be negligible for PWR primary-water-chemistry conditions and for BWRs that add hydrogen to the feedwater (HWC) and attain water chemistries that meet the purity and electrochemical potential (ECP) conditions set forth in Ref. 3. The air term, based on the work of James and Jones, ${ }^{4}$ is given by the current ASME Section XI correlation at $288^{\circ} \mathrm{C}$ as 


$$
\begin{array}{rlrl}
\dot{\mathbf{a}}_{\text {air }} & =3.43 \times 10^{-12} \mathrm{~S}(\mathrm{R}) \Delta \mathrm{K}^{3.3} / \mathrm{T}_{\mathrm{R}}\left(\mathrm{m} \cdot \mathrm{s}^{-1}\right) \\
\mathrm{S}(\mathrm{R}) & =1+1.18 \mathrm{R} & & \mathrm{R} \leq 0.8 \\
& =-43.35+57.97 \mathrm{R} & & \mathrm{R}>0.8
\end{array}
$$

where $T_{R}$ is the rise time (s) of the loading wave form, $R$ is the load ratio $\left(K_{\min } / K_{\max }\right)$, and $\Delta \mathrm{K}$ is $\mathrm{K}_{\max }-\mathrm{K}_{\mathrm{min}}$. Following Shoj $1^{5}$ and Gilman et al., ${ }^{2}$ the corrosion fatigue term is assumed to be related to $\dot{a}_{\text {air }}$ through a power law.

$$
\dot{\mathbf{a}}_{\text {env }}=\mathbf{A} \dot{a}_{\text {air }}^{m} \text {. }
$$

The values of the coefficient $A$ and the exponent $m$ for water with $200 \mathrm{ppb}$ DO at $288^{\circ} \mathrm{C}$ were obtained by an empirical power-law-curve fit to the existing data for $R<0.9$, where cyclic loading dominates and the stress corrosion term in the superposition model (Eq. 1) can be ignored. The values are

$$
\begin{aligned}
& A=4.5 \times 10^{-5} \\
& \mathrm{~m}=0.5,
\end{aligned}
$$

for CGRs in $\mathrm{m} \cdot \mathrm{s}^{-1}$ and $\mathrm{K}$ in $\mathrm{MPa} \cdot \mathrm{m}^{1 / 2}$.

In water with $8 \mathrm{ppm} \mathrm{DO}$ at $288^{\circ} \mathrm{C}$, an empirical power-law-curve fit to the avallable data gives

$$
\begin{aligned}
& \mathrm{A}=1.5 \times 10^{-4} \\
& \mathrm{~m}=0.5,
\end{aligned}
$$

for CGRs in $\mathrm{m} \cdot \mathrm{s}^{-1}$ and $\mathrm{K}$ in $\mathrm{MPa} \cdot \mathrm{m}^{1 / 2}$.

Figures 1 and 2 show a comparison of most of the avallable experimental data at $288^{\circ} \mathrm{C}$ for sensitized Type 304 SS in water wilh 0.2 and 8 ppm DO, respectively, with correlations based on Eqs. 1-6. Data for Types 316NG and solution-annealed 304 SS (all nonsensitized) are compared with that for sensilized SS in Fig. 3 . Although it is clear that the nonsensitized steels are much more resistant to SCC initiation, they show comparable environmentally enhanced CGRs under cyclic loading. This is also true for cast SSs in the as-received and thermally aged conditions in water with 0.2 and $8 \mathrm{ppm}$ DO, shown in Figs. 4 and 5 , respectively. At DO concentrations $>1 \mathrm{ppm}$, the CGRs of thermally aged CF-8M are higher by one order of magnitude than CGRs for the steel in the as-recelved condition. Thermal aging has a smaller elfect on CGRs of CF-8 SS under these conditions.

In most cases, the correlations were intended to be conservative but not necessarlly upper bounds for all of the data. The data from the ANL tests on wrought SSs in simulated BWR water have been reported in the series of ANL semlannual reports on the NRCsponsored program and are summari\%ed in Rel. 6. Data on as-recelved and thermally aged cast SSs were reported in Ref. 7. Other data were obtained from the literature (Refs. 8-14).

Ford et al. ${ }^{15}$ developed a CGR model that includes the effects of DO concentration (through changes in ECP). The model suggests that environmentally enhanced CGRs (as determined by coefficient $A$ in the current case) should decrease by at least one crder of magnitude when the DO concentration decreases from $\approx 8$ to $0.2 \mathrm{ppm}$. Based on slowstrain-rate-tensile ( SSRT) tests, Kassner et al. ${ }^{16}$ suggested that the dependence of CGR on DO concentration $\left[\mathrm{O}_{2}\right]$ follows an $\approx\left[\mathrm{O}_{2}\right]^{1 / 4}$ relationship over this range of oxygen concentrations, 1.e., the rates are somewhat less dependent on oxygen concentration. The empirically determined decrease in $A$, corresponding to a decrease in DO from 8 to $0.2 \mathrm{ppm}$ 
(Eqs. 5 and 6), is in reasonable agreement with that predicted in Ref. 16. It is $\approx 1 / 3$ of the decrease predicted by the model of Ref. 15. Both models predict that the environmental contribution to the CGR should continue to decrease with further reductions in DO or ECP. However, as shown in Fig. 6 (also note the data of Kawakubo in Fig. 7), the limited data at lower $R$ values and low DO concentrations $(<20 \mathrm{ppb})$ are consistent with the superposition model, Eq. 1, when the term corresponding to SCC (Eq. 2) is deleted, (namely, the curves denoted as $\dot{a}_{\text {super }} \mathrm{w} / \mathrm{o} \mathrm{SCC}$ ) with the value of $\mathrm{A}$ determined from data obtained in water with $0.2 \mathrm{ppm}$ DO. Hence, in the present model, we assume that low DO and ECP levels associated with HWC eliminate the SCC contribution in the superposition model Eq. (1), but that the corrosion fatigue term, a env, given by Eq. (4), is still important. As shown in Fig. 6, the model of Ford et al.,15 which predicts a decrease in the value of $A$ in water with low DO content, appears to be somewhat nonconservative with respect to the available data.
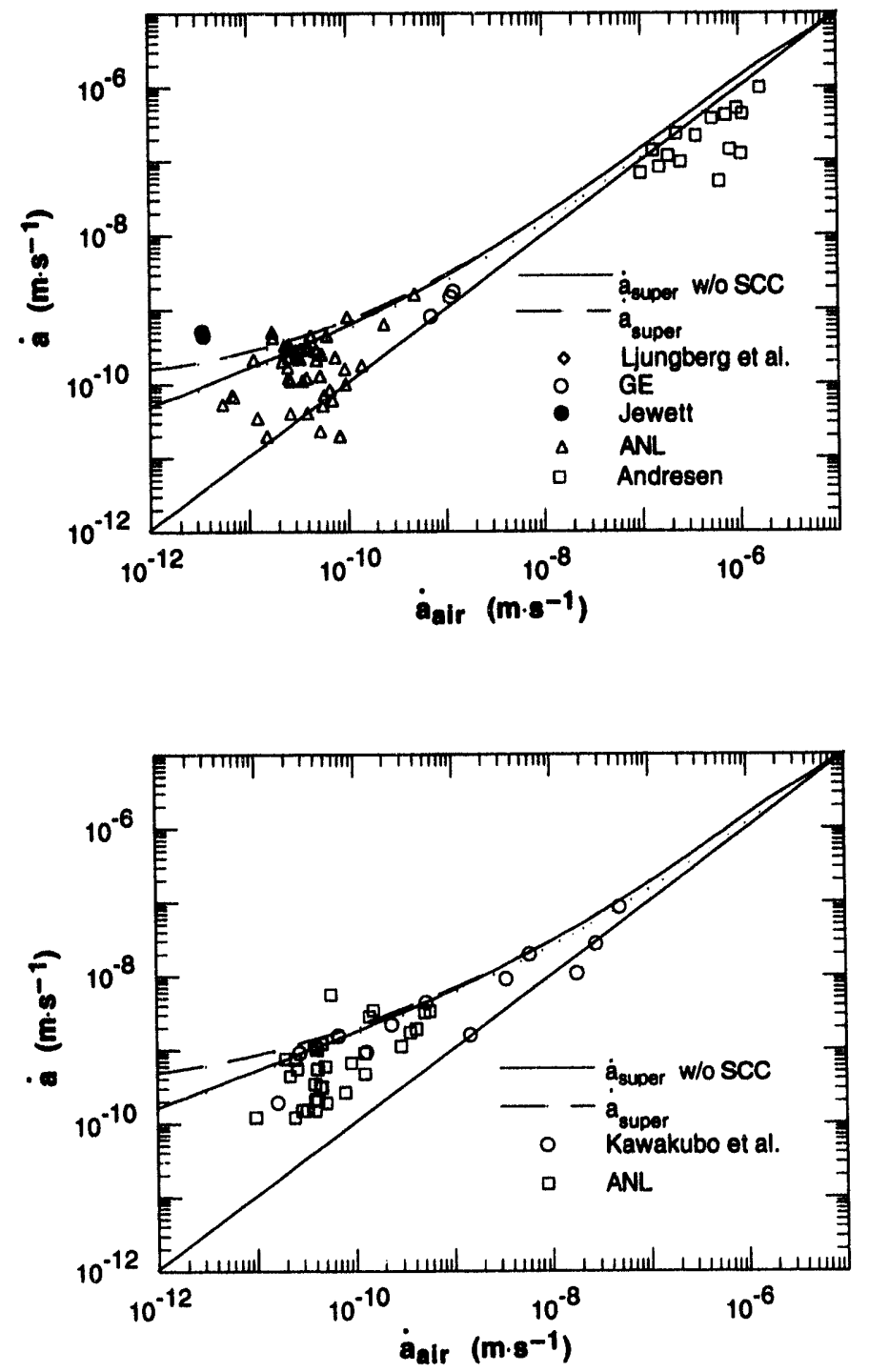

Fig. 1.

Corrosion fatigue data for wrought SSs in water containing 200 ppb DO at $288^{\circ} \mathrm{C}$ compared with models. Diagorial line corresponds to crack growth in air.

Fig. 2.

Corrosion fatigue data for wrought SSs in water containing 8 ppm DO at $288^{\circ} \mathrm{C}$ compared with modeis. Diagonal line corresponds to crack growth in air. 

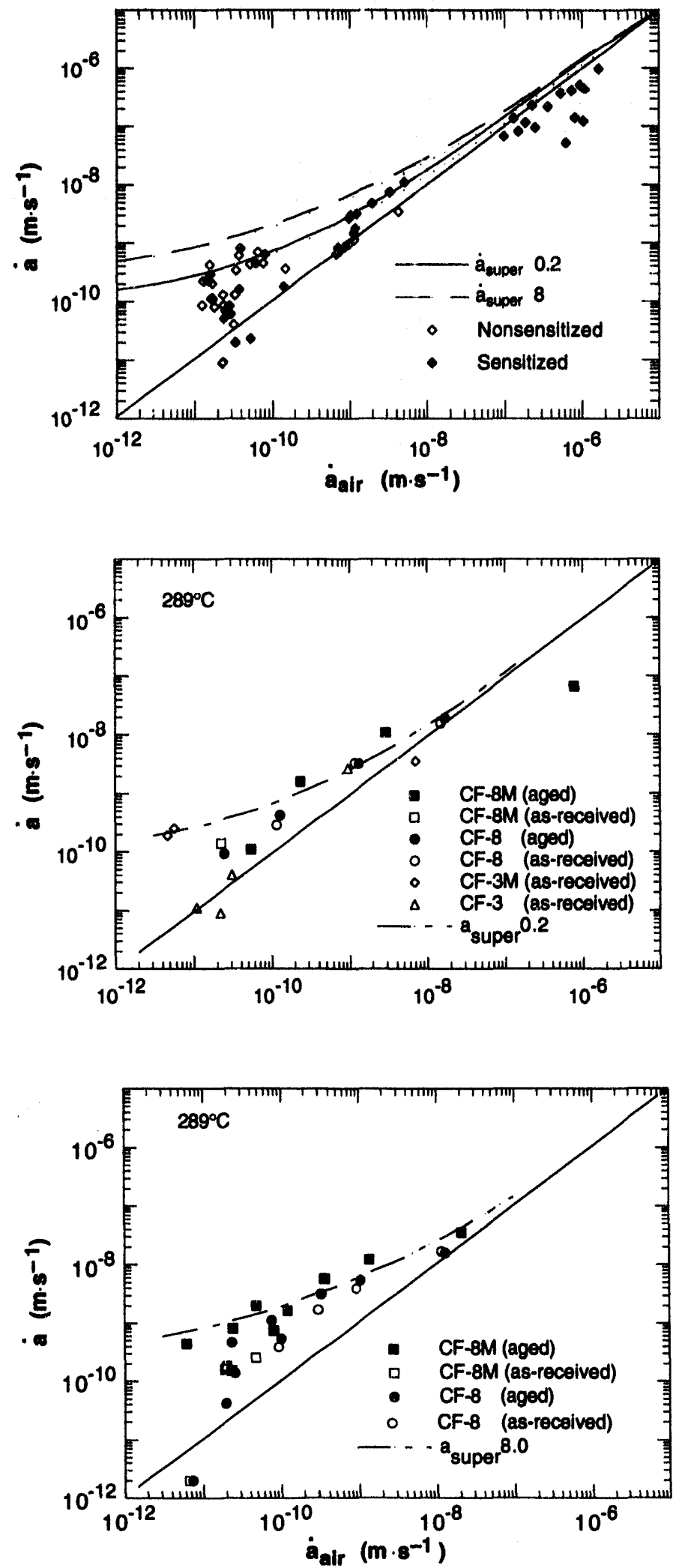

Fig. 3.

Corrosion fatigue data for sensittzed and nonsensitized SSs in water containing $200 \mathrm{ppb}$ DO at $288^{\circ} \mathrm{C}$. Diagonal line corresponds to crack growth in air.

Flg. 4.

Corrosion fatigue data for cast SSs in water containing 200 ppb DO at $289^{\circ} \mathrm{C}$ compared with model. Diagonal line corresponds to crack growth of wrought SSs in air.
Fig. 5.

Corrosion fatigue data for cast SSs in water containing $8 \mathrm{ppm} \mathrm{DO}$ at $289^{\circ} \mathrm{C}$ compared with model. Diagonal line corresponds to crack growth of wrought SSs in air. 

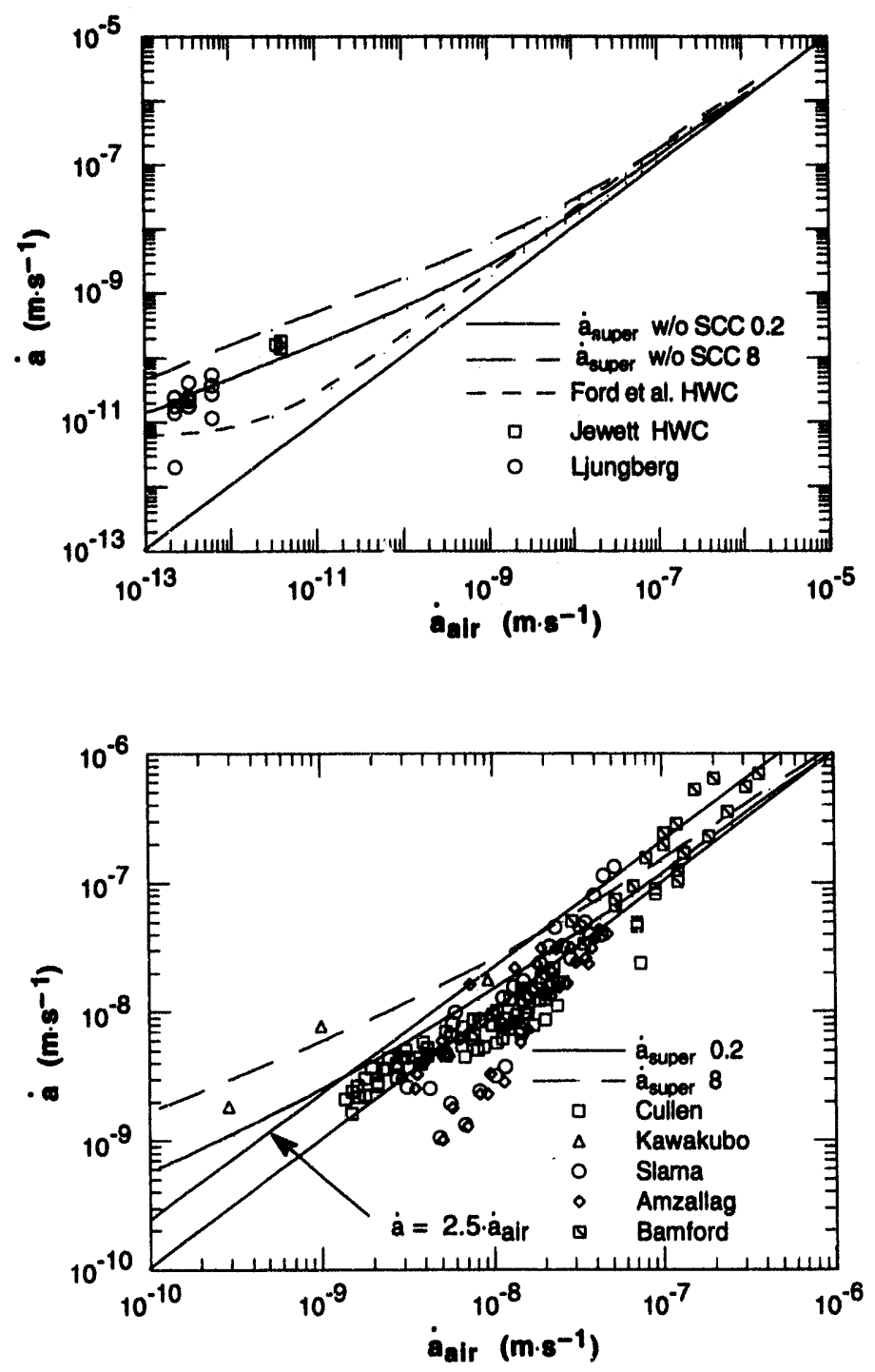

Fig. 6 .

Corrosion fatigue data for wrought SSs in water containing low DO at $288^{\circ} \mathrm{C}$, superposition models, and a model of Ford et al. 15 Curve for Ford et al. assumes an ECP of $-250 \mathrm{mV}$ (SHE), a conductivity of $0.25 \mu \mathrm{S} \cdot \mathrm{cm}^{-1}$ and an EPR of $15 \mathrm{C} \cdot \mathrm{cm}^{-2}$. Diagonal line corresponds to crack growth in air.

Fig. 7. Corrosion fatigue data for wrought SSs in simulated PWR water and curves from models based on data obtained in simulated BWR water. Diagonal line corresponds to crack growth in air.

Data obtained from Refs. 17-20 for simulated PWR water and unpublished data by Kawakubo are shown in Fig. 7, along with the corrosion-fatigue curves developed for water with 0.2 and $8 \mathrm{ppm}$ DO. Also shown in Fig. 7 is a correlation, similar to that proposed by Bernard and Slama, 21 which increases the CGR in air by a factor of 2.5. This curve bounds almost all of the data, except for those of Kawakubo. However, all data (except for Kawakubo's) represent rather high CGRs $\left(<10^{-9} \mathrm{~m} \cdot \mathrm{s}^{-1}\right)$. Until additional data become avallable at lower frequencies in simulated PWR water, it seems prudent, when extrapolating to lower CGRs, to use the curve based on the data obtained in water with $200 \mathrm{ppb}$ DO, but which does not include an SCC term, because SCC is very unlikely in PWR water with low DO content.

The correlations proposed here are very similar to those proposed by Gilman et al.,2 ${ }^{2}$ i.e., one for water containing $8 \mathrm{ppm}$ DO and another for nonsenstized Type 316NG SS in water

\footnotetext{
T. Kawakubo, Accs. 102486, 102487, EDEAC Database, Battelle Columbus. Obtained by private communication from E. Eason, Modeling \& Computer Services, April 1991.
} 
containing $200 \mathrm{ppb}$ DO. However, the correlation proposed here for water containing $200 \mathrm{ppb}$ DO is assumed to be applicable to both sensitized and nonsensitized SSs. The predictions of the models developed in Ref. 2 and those presented here are shown in Fig. 8. The differences are very small, and superposition of an SCC model with the model of Gilman et al. 2 eliminates any significant differences.
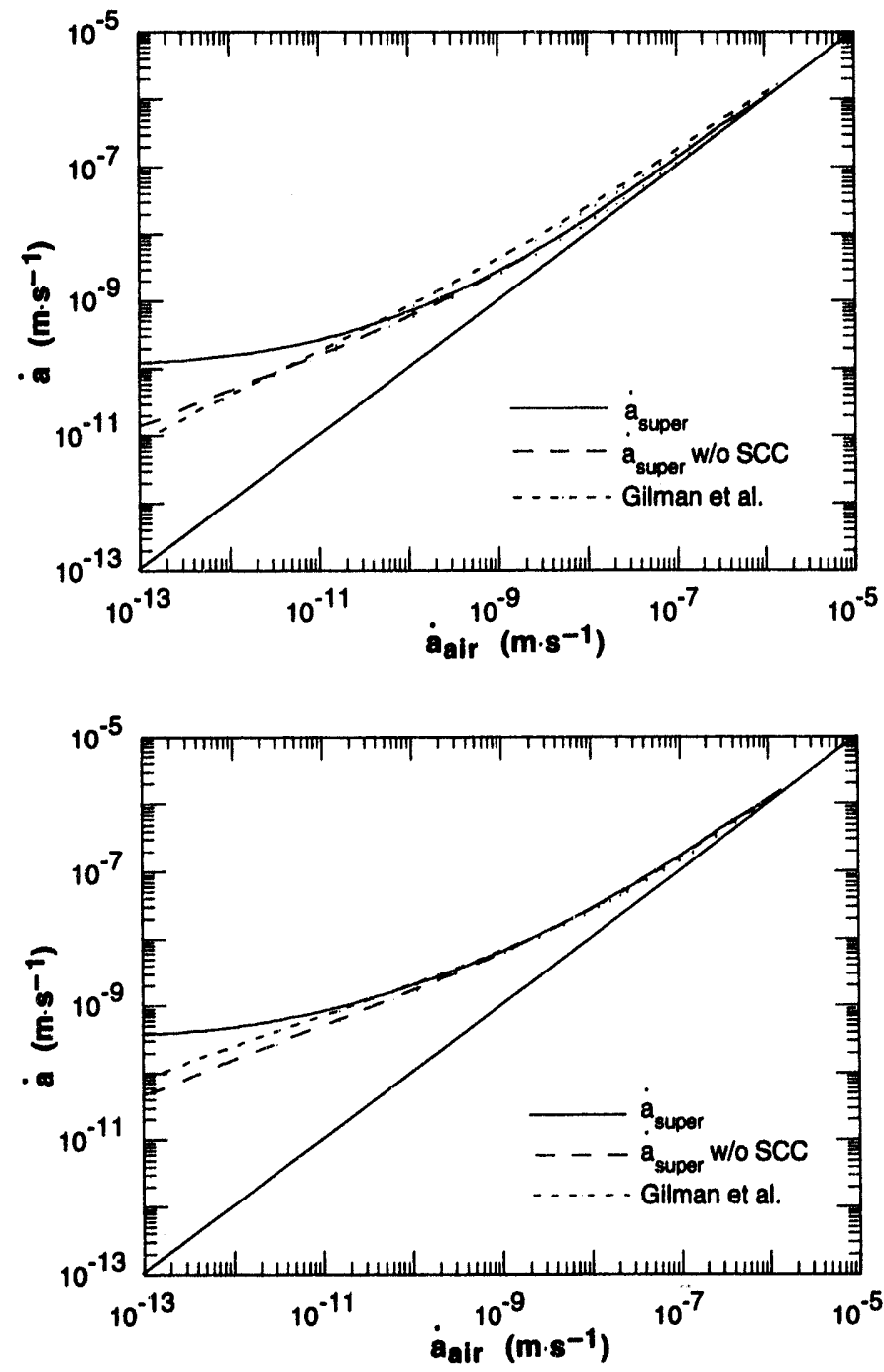

Fig. 8a.

Current model and model proposed by Gilman et al. 2 in water containing 200 ppb DO.

Diagonal line corresponds to crack growth of wrought SSs in air.

Fig. $8 b$.

Current model and model proposed by Gilman et al. 2 in water containing 8 ppm DO. Diagonal line corresponds to crack growth of wrought SSs in air.

The correlations and data presented here have focused on water at $288^{\circ} \mathrm{C}$. Relatively few data are avallable in the literature on corrosion-fatigue CGRs at other temperatures. SSRT data by Ruther et al. 22 and Ford ${ }^{23}$ suggest that environmentally assisted CGRs peak at $\approx 200-225^{\circ} \mathrm{C}$ and that in high-purity (HP) water (conductivity $<0.2 \mu \mathrm{S} \cdot \mathrm{cm}^{-1}$ ), CGRs decrease at both higher and lower temperatures. In tests by Ruther et al.22 at higher impurity levels (conductivity $>0.9 \mu \mathrm{S} \cdot \mathrm{cm}^{-1}$ ), no decrease was observed at higher temperatures. Because the tests were performed only with $\mathrm{H}_{2} \mathrm{SO}_{4}$ additions, it is not known whether this effect is associated with specific chemical species or depends only on the overall impurity (conductivity) level. In fracture-mechanics CGR tests at $R=0.95$, Ruther et al. 24 confirmed that in $\mathrm{HP}$ water, CGRs at $320^{\circ} \mathrm{C}$ were much lower than at $288^{\circ} \mathrm{C}$. Andresen 25 found that CGRs in fracture-mechanics specimens of sensitized Type 304 SS were higher by a factor of $10-20$ at $200^{\circ} \mathrm{C}$ than at $288^{\circ} \mathrm{C}$ and the data also suggest a sharp drop in CGRs at temperatures $>300^{\circ} \mathrm{C}$ in $\mathrm{HP}$ water. 
For convenience, the equations needed to describe corrosion fatigue in aqueous environments are summarized in Table 1 . In most cases, one of the terms in the superposition models is dominant, but the magnitude of each of the terms should be estimated before a simplified expression is used. Alternatively, because in most cases $T_{R}$ and $R$ are fixed, the CGR at the lowest $\Delta K$ of interest can be computed and compared to the corresponding CGR in air. This computation will determine a conservative multiplier that can be applied to a CGR (or da/dN curve) in air for subsequent calculations. The equations for $\dot{a}_{a i r}$ and $\hat{a}_{\text {env }}$ can be rewritten in the more familiar da/dN form, as shown in Table 2. However, as in the case of the new curves for ferritic steels proposed for Section $X I$ of the ASME code, the cyclic crack growth curves in water are dependent on the rise time (frequency).

Table 1. Summary of equations for corrosion fatigue in BWR and PWR environments $a$

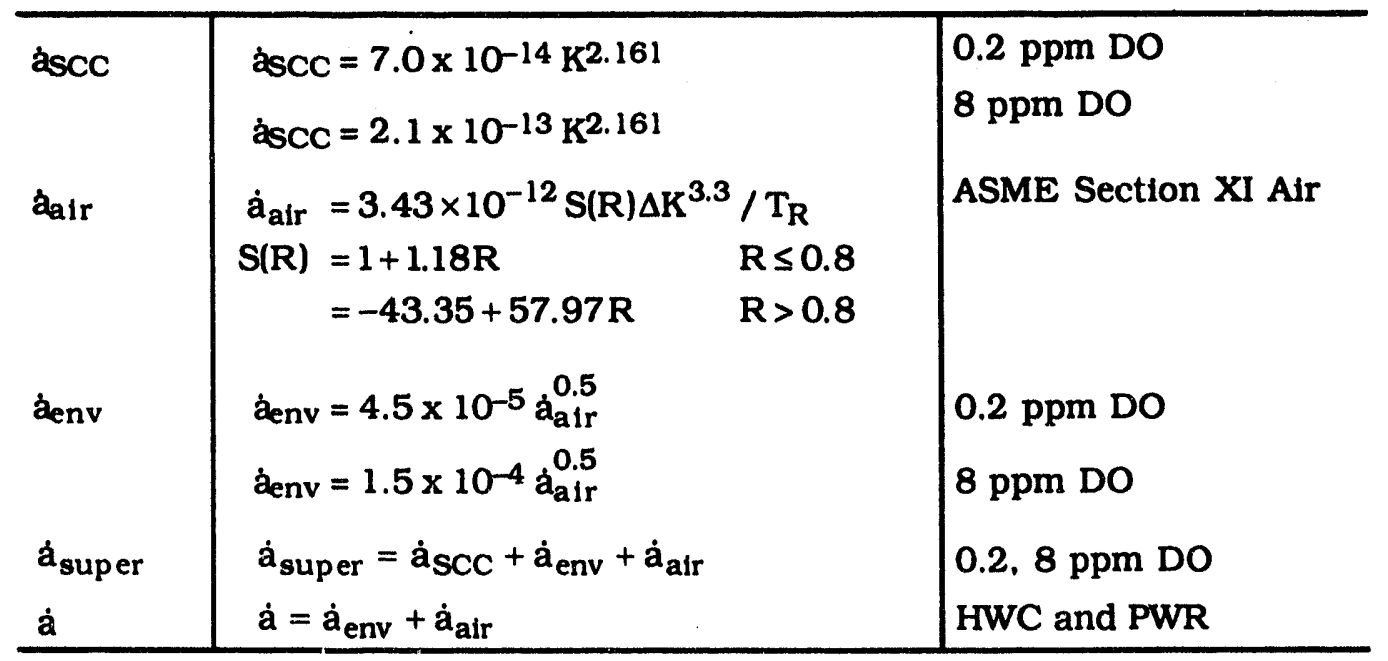

aAll crack growth rates in $\mathrm{m} \cdot \mathrm{s}^{-1}, \mathrm{~K}$ in $\mathrm{MPa} \cdot \mathrm{m}^{1 / 2}$, and $\mathrm{T}_{\mathrm{R}}$ in $\mathrm{s}$.

Table 2. Corrosion fatigue curves in BWR and PWR environments in $d a / d N$ form $a$

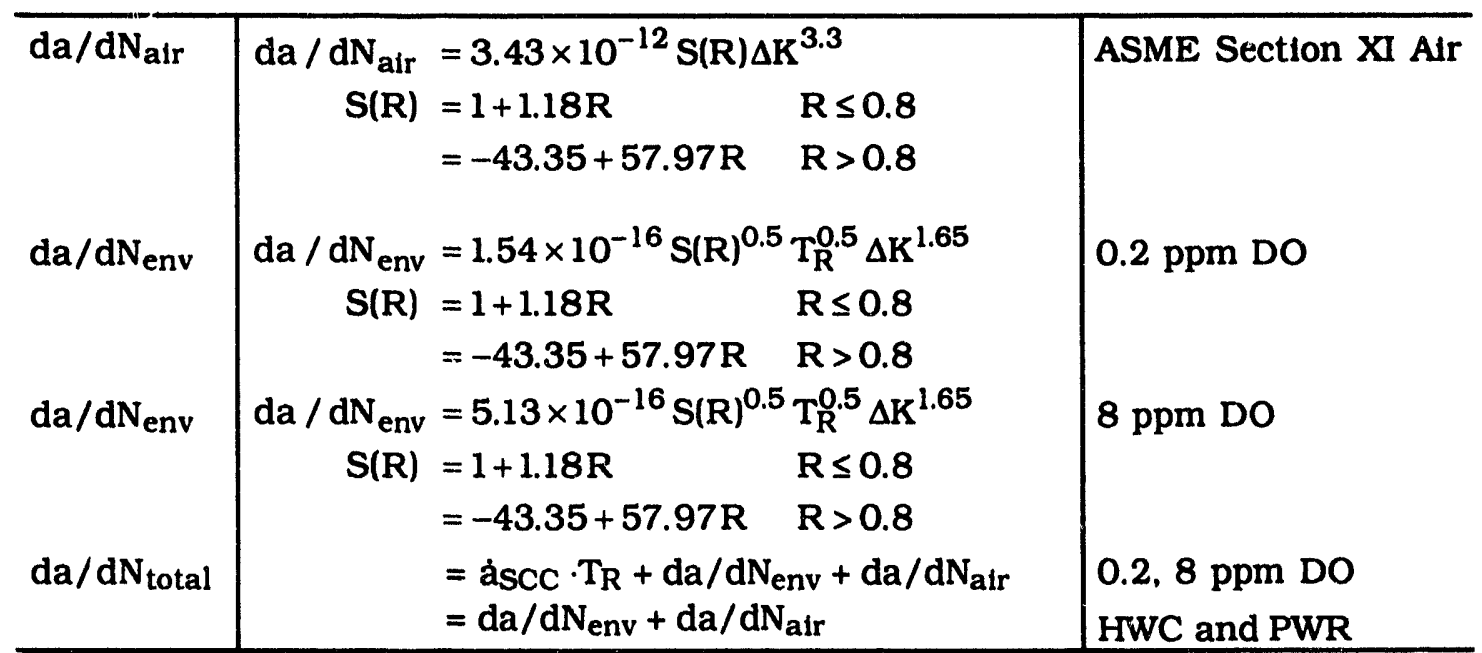

aAll crack growth rates in $m \cdot$ cycle $^{-1}, \mathrm{~K}$ in $\mathrm{MPa} \cdot \mathrm{m}^{1 / 2}$, and $\mathrm{T}_{\mathrm{R}}$ in $\mathrm{s}$. 


\section{Appendix A: ANL Crack Growth Data Base for Wrought and Cast Austenitic Stainless Steels}

Crack growth tests have been conducted on fracture-mechanics specimens of wrought Types 304, 316NG, and 347 and cast CF-3, CF-3M, CF-8, and CF-8M SSs to characterize the environmental, loading, and material conditions that can produce SCC susceptibility in these steels. In the case of the cast SSs. CGR data were obtained on material in the as-recetved and thermally aged $\left(10,000 \mathrm{~h}\right.$ at $400^{\circ} \mathrm{C}$ and $30,000 \mathrm{~h}$ at $\left.350^{\circ} \mathrm{C}\right)$ conditions. Data that have been obtained over the past 10 years (October 1983 to September 1993) are summarzed in Refs. 6 and 7, along with references that contain details of the test methods, composition of the materials, metallographic and fractographic information. Comparisons of the data with predictions based on the corrosion-fatigue models given in Table 1 were also presented. Agreement between predictions of the models and data for cast SSs was good.

Crack growth results for Type $316 \mathrm{NG}$ SS and sensitized $(E P R=2,8,20$, and $30 \mathrm{C} \cdot \mathrm{cm}^{-2}$ ) and solution-annealed Type $304 \mathrm{SS}$ in HP water containing $\approx 200 \mathrm{ppb}$ DO at $289^{\circ} \mathrm{C}$ are summarized in Table 3 . Most of the data were obtained under high-R (0.8-0.95), low-frequency $\left(8 \times 10^{-2} \mathrm{~Hz}\right)$ loading conditions at maximum stress intensity values of 27-46 MPa $\mathrm{m}^{1 / 2}$. Table 4 contains data on sensilized and solution-annealed Type $304 \mathrm{SS}$ in HP water containing 5-8 ppm DO at $289^{\circ} \mathrm{C}$ over a wider range of load ratio, frequency, and stress intensity. Crack growth results for Type $316 \mathrm{NG}$ and sensitized and solution-annealed Type $304 \mathrm{SS}$ at $289^{\circ} \mathrm{C}$ in water containing $\approx 200 \mathrm{ppb}$ DO and ionic impurities (namely, $\mathrm{SO}_{4}^{2-}$. $\mathrm{CrO}_{4}^{2-} \cdot \mathrm{NO}_{3}^{-}$, and carboxylic acids) are summarized in Table 5. Most of the data were obtained at a load ratio of 0.95 and a frequency of $8 \times 10^{-2} \mathrm{~Hz}$. The influence of several impurity species at concentrations of $\approx 6-1000 \mathrm{ppb}$ (conductivity values $\approx 0.2-3.7 \mu \mathrm{S} \cdot \mathrm{cm}^{-1}$ ) on CGRs in the two steels can be obtained from these data. The effect of degree of sensitization corresponding to EPR values of 0-30 C.cm-2 on CGRs in Type 304 SS can also be determined from these data. Table 6 summarizes CGR results for Type 347 SS specimens with different heat treatment conditions (slow-cooled and water quenched from the austenitizing temperature). These data were obtained in water containing $=200 \mathrm{ppb} D O$ and $100 \mathrm{ppb} \mathrm{SO}_{4}^{2-}$ at $289^{\circ} \mathrm{C}$. Table 7 gives the CGR results on specimens of $\mathrm{CF}-3, \mathrm{CF}-3 \mathrm{M}$, $\mathrm{CF}-8$, and $\mathrm{CF}-8 \mathrm{M}$ grades of cast SS in the as-received and thermally aged conditions. 
Table 3. Summary of crack growth results for Type 316NG and sensitized and solution-annealed Type 304 SS specimensa in oxygenated $(=200 \mathrm{ppb})$ waterb at $289^{\circ} \mathrm{C}$, in which load ratioc and stress intensity were varied

\begin{tabular}{|c|c|c|c|c|c|c|c|c|c|c|c|c|c|c|}
\hline \multirow[b]{2}{*}{$\begin{array}{l}\text { Cond., } \\
\mu \mathrm{S} \cdot \mathrm{cm}^{-1}\end{array}$} & \multicolumn{2}{|c|}{ Potentials } & \multirow{2}{*}{$\begin{array}{l}\text { Load } \\
\text { Ratio }\end{array}$} & \multirow[b]{2}{*}{$\begin{array}{l}\text { Freq.. } \\
10^{-2} \mathrm{~Hz}\end{array}$} & \multirow{2}{*}{$\begin{array}{c}\text { Rise } \\
\text { Time, } \\
\text { s }\end{array}$} & \multicolumn{3}{|c|}{ Type 316NG SS } & \multicolumn{4}{|c|}{ Type 304 SS } & \multirow[b]{2}{*}{$\begin{array}{l}\text { Ref. } \\
\text { No. }\end{array}$} & \multirow{2}{*}{$\begin{array}{l}\text { ANL } \\
\text { Report } \\
\text { No. }\end{array}$} \\
\hline & $\begin{array}{l}304 \text { SS. } \\
\text { mVISHF }\end{array}$ & Pt. & & & & $\begin{array}{l}\overline{K_{\text {max }}} \text { d. } \\
\text { MPa. }\end{array}$ & $\Delta \mathrm{K}, \mathrm{e}$ & $\begin{array}{l}\text { Rate, } \\
10^{-10} \mathrm{~m} \cdot \mathrm{s}^{-1}\end{array}$ & $\begin{array}{r}\mathrm{K}_{\max }{ }^{\mathrm{d}} \\
\mathrm{MPa}\end{array}$ & $\Delta K$ & $\begin{array}{c}\text { Rate, } \\
10^{-10} \mathrm{~m} \cdot \mathrm{s}^{-1}\end{array}$ & $\begin{array}{l}\mathrm{EPR}, \\
\mathrm{C \textrm {cm } ^ { - 2 }}\end{array}$ & & \\
\hline $\begin{array}{l}0.11 \\
0.11 \\
0.11\end{array}$ & $\begin{array}{l}\overline{-} \\
\overline{-}\end{array}$ & $\overline{-}$ & $\begin{array}{l}0.95 \\
0.95 \\
0.95\end{array}$ & $\begin{array}{l}8 \\
8 \\
8\end{array}$ & $\begin{array}{l}12 \\
12 \\
12\end{array}$ & $\begin{array}{l}- \\
\overline{-}\end{array}$ & $\bar{z}$ & $\overline{\bar{z}}$ & $\begin{array}{l}27 . \overline{3} \\
29.3 \\
29.9\end{array}$ & $\begin{array}{l}1.37 \\
1.46 \\
1.50\end{array}$ & $\begin{array}{l}1.00 \\
3.30 \\
3.00\end{array}$ & $\begin{array}{r}0 \\
2 \\
20\end{array}$ & $\begin{array}{l}49 \\
49 \\
49\end{array}$ & $\begin{array}{l}83-85 \text { IV } \\
83-85 \text { IV } \\
83-85 \text { IV }\end{array}$ \\
\hline $\begin{array}{l}0.16 \\
0.16 \\
0.16\end{array}$ & $\begin{array}{l}- \\
-\end{array}$ & $\overline{-}$ & $\begin{array}{l}0.95 \\
0.95 \\
0.95\end{array}$ & $\begin{array}{l}8 \\
8 \\
8\end{array}$ & $\begin{array}{l}12 \\
12 \\
12\end{array}$ & $\begin{array}{l}- \\
-\end{array}$ & $\overline{-}$ & $\begin{array}{l}- \\
-\end{array}$ & $\begin{array}{l}28.0 \\
30.3 \\
31.3\end{array}$ & $\begin{array}{l}1.40 \\
1.51 \\
1.56\end{array}$ & $\begin{array}{l}1.80 \\
0.40 \\
2.80\end{array}$ & $\begin{array}{r}0 \\
2 \\
20\end{array}$ & $\begin{array}{l}49 \\
49 \\
49\end{array}$ & $\begin{array}{l}83-85 \text { IV } \\
83-85 \text { IV } \\
83-85 \text { IV }\end{array}$ \\
\hline $\begin{array}{l}0.14 \\
0.14 \\
0.14\end{array}$ & $\begin{array}{l}- \\
-\end{array}$ & $\begin{array}{l}- \\
-\end{array}$ & $\begin{array}{l}0.95 \\
0.95 \\
0.95\end{array}$ & $\begin{array}{l}8 \\
8 \\
8\end{array}$ & $\begin{array}{l}12 \\
12 \\
12\end{array}$ & $\begin{array}{l}- \\
-\end{array}$ & - & $\begin{array}{l}- \\
-\end{array}$ & $\begin{array}{l}28.4 \\
31.5 \\
31.9\end{array}$ & $\begin{array}{l}1.42 \\
1.57 \\
1.59\end{array}$ & $\begin{array}{l}1.60 \\
2.50 \\
2.20\end{array}$ & $\begin{array}{r}0 \\
2 \\
20\end{array}$ & $\begin{array}{l}49 \\
49 \\
49\end{array}$ & $\begin{array}{l}83-85 \text { IV } \\
83-85 \text { IV } \\
83-85 \text { IV }\end{array}$ \\
\hline $\begin{array}{l}0.15 \\
0.15 \\
0.15 \\
0.15 \\
0.15\end{array}$ & $\begin{array}{l}- \\
- \\
- \\
-\end{array}$ & $\begin{array}{l}- \\
- \\
- \\
-\end{array}$ & $\begin{array}{l}0.95 \\
0.95 \\
0.95 \\
0.95 \\
0.95\end{array}$ & $\begin{array}{l}8 \\
8 \\
8 \\
8 \\
8\end{array}$ & $\begin{array}{l}12 \\
12 \\
12 \\
12 \\
12\end{array}$ & $\begin{array}{l}- \\
- \\
- \\
-\end{array}$ & $\begin{array}{l}- \\
\overline{-} \\
- \\
-\end{array}$ & $\begin{array}{l}- \\
- \\
- \\
-\end{array}$ & $\begin{array}{l}30.0 \\
37.0 \\
38.0 \\
37.0 \\
37.0\end{array}$ & $\begin{array}{l}1.50 \\
1.85 \\
1.90 \\
1.85 \\
1.85\end{array}$ & $\begin{array}{l}1.70 \\
2.50 \\
0.84 \\
2.10 \\
0.51\end{array}$ & $\begin{array}{r}0 \\
2 \\
2 \\
20 \\
20\end{array}$ & $\begin{array}{l}\mathbf{5 0} \\
\mathbf{5 0} \\
\mathbf{5 0} \\
\mathbf{5 0} \\
\mathbf{5 0}\end{array}$ & $\begin{array}{l}85-33 \\
85-33 \\
85-33 \\
85-33 \\
85-33\end{array}$ \\
\hline $\begin{array}{l}0.10 \\
0.10 \\
0.10 \\
0.10\end{array}$ & $\begin{array}{l}138 \\
115 \\
145 \\
140\end{array}$ & $\begin{array}{l}219 \\
230 \\
220 \\
210\end{array}$ & $\begin{array}{l}0.95 \\
0.95 \\
0.95 \\
0.95\end{array}$ & $\begin{array}{l}8 \\
8 \\
8 \\
8\end{array}$ & $\begin{array}{l}12 \\
12 \\
12 \\
12\end{array}$ & $\begin{array}{l}31.2 \\
33.7 \\
34.1 \\
34.6\end{array}$ & $\begin{array}{l}1.56 \\
1.68 \\
1.70 \\
1.73\end{array}$ & $\begin{array}{l}1.40 \\
2.10 \\
0.50 \\
0.40\end{array}$ & $\begin{array}{l}33.6 \\
34.9 \\
36.2 \\
37.1\end{array}$ & $\begin{array}{l}1.68 \\
1.75 \\
1.81 \\
1.86\end{array}$ & $\begin{array}{l}0 \\
0 \\
1.30 \\
0.70\end{array}$ & $\begin{array}{l}2 \\
2 \\
2 \\
2\end{array}$ & $\begin{array}{l}51 \\
511 \\
51 \\
51\end{array}$ & $\begin{array}{l}87-37 \\
87-37 \\
87-37 \\
87-37\end{array}$ \\
\hline 0.21 & 95 & 110 & 0.95 & 8 & 12 & - & - & - & 29.7 & 1.49 & 1.70 & 2 & 52 & $87-41$ \\
\hline $\begin{array}{l}0.13 \\
0.12 \\
0.15\end{array}$ & $\begin{array}{r}75 \\
80 \\
140\end{array}$ & $\begin{array}{r}8 \overline{5} \\
160\end{array}$ & $\begin{array}{l}0.95 \\
0.95 \\
0.95\end{array}$ & $\begin{array}{l}8 \\
8 \\
8\end{array}$ & $\begin{array}{l}12 \\
12 \\
12\end{array}$ & $\begin{array}{l}32.1 \\
32.2 \\
33.2\end{array}$ & $\begin{array}{l}1.61 \\
1.61 \\
1.66\end{array}$ & $\begin{array}{l}3.00 \\
0.25 \\
1.10\end{array}$ & $\begin{array}{l}- \\
-\end{array}$ & $\begin{array}{l}- \\
-\end{array}$ & $\begin{array}{l}- \\
-\end{array}$ & $\overline{-}$ & $\begin{array}{l}\mathbf{5 3} \\
\mathbf{5 3} \\
\mathbf{5 3}\end{array}$ & $\begin{array}{l}88-32 \\
88-32 \\
88-32\end{array}$ \\
\hline $\begin{array}{l}0.20 \\
0.20\end{array}$ & $\begin{array}{l}170 \\
170\end{array}$ & $\begin{array}{l}190 \\
205\end{array}$ & $\begin{array}{l}0.95 \\
0.95\end{array}$ & $\begin{array}{l}8 \\
8\end{array}$ & $\begin{array}{l}12 \\
12\end{array}$ & $\begin{array}{l}30.5 \\
33.0\end{array}$ & $\begin{array}{l}1.53 \\
1.65\end{array}$ & $\begin{array}{l}2.20 \\
2.00\end{array}$ & $\begin{array}{l}28.8 \\
29.9\end{array}$ & $\begin{array}{l}1.44 \\
1.50\end{array}$ & $\begin{array}{l}2.00 \\
1.20\end{array}$ & $\begin{array}{l}2 \\
2\end{array}$ & $\begin{array}{l}54 \\
54\end{array}$ & $\begin{array}{l}89-10 \\
89-10\end{array}$ \\
\hline 0.11 & 165 & 132 & 1.00 & 0 & $\infty$ & 24.5 & 0.00 & 0.27 & 24.5 & 0.00 & 0.53 & 2 & 55 & $89-40$ \\
\hline 0.19 & 167 & 190 & 1.00 & 0 & $\infty$ & 29.0 & 0.00 & 0.34 & 26.0 & 0.00 & 0.68 & 2 & 55 & $89-40$ \\
\hline $\begin{array}{l}0.12 \\
0.12 \\
0.11 \\
0.13 \\
0.16\end{array}$ & $\begin{array}{l}112 \\
125 \\
145 \\
157 \\
182\end{array}$ & $\begin{array}{l}182 \\
195 \\
175 \\
152 \\
190\end{array}$ & $\begin{array}{l}0.95 \\
0.95 \\
0.95 \\
0.95 \\
0.95\end{array}$ & $\begin{array}{l}\mathbf{8} \\
\mathbf{8} \\
\mathbf{8} \\
\mathbf{8} \\
\mathbf{8}\end{array}$ & $\begin{array}{l}12 \\
12 \\
12 \\
12 \\
12\end{array}$ & $\begin{array}{l}19.8 \\
22.6 \\
24.4 \\
24.6 \\
29.0\end{array}$ & $\begin{array}{l}0.96 \\
1.13 \\
1.22 \\
1.23 \\
1.45\end{array}$ & $\begin{array}{l}\approx 0 \\
0.63 \\
1.40 \\
0.11 \\
0.41\end{array}$ & $\begin{array}{l}17.6 \\
21.2 \\
24.2 \\
24.7 \\
26.1\end{array}$ & $\begin{array}{l}0.87 \\
1.06 \\
1.21 \\
1.23 \\
1.30\end{array}$ & $\begin{array}{l}\approx 0 \\
0.70 \\
2.10 \\
0.35 \\
0.20\end{array}$ & $\begin{array}{l}2 \\
2 \\
2 \\
2 \\
2\end{array}$ & $\begin{array}{l}\mathbf{5 5} \\
\mathbf{5 5} \\
\mathbf{5 5} \\
\mathbf{5 5} \\
\mathbf{5 5}\end{array}$ & $\begin{array}{l}89-40 \\
89-40 \\
89-40 \\
89-40 \\
89-40\end{array}$ \\
\hline
\end{tabular}




\section{Table 3. Continued}

\begin{tabular}{|c|c|c|c|c|c|c|c|c|c|c|c|c|c|c|}
\hline \multirow{3}{*}{$\begin{array}{l}\text { Cond., } \\
\mu \mathrm{S} \cdot \mathrm{cm}^{-1} \\
0.16 \\
0.13 \\
0.12 \\
0.11 \\
0.15\end{array}$} & \multicolumn{2}{|c|}{ Potentials } & \multirow{2}{*}{$\begin{array}{l}\text { Load } \\
\text { Ratio }\end{array}$} & \multirow[b]{2}{*}{$\begin{array}{l}\text { Freq., } \\
10^{-2} \mathrm{~Hz}\end{array}$} & \multirow{2}{*}{$\begin{array}{c}\text { Rise } \\
\text { Time, } \\
\text { s }\end{array}$} & \multicolumn{3}{|c|}{ Type 316NG SS } & \multicolumn{4}{|c|}{ Type 304 SS } & \multirow[b]{2}{*}{$\begin{array}{ll} & \text { Ref. } \\
2 & \text { No. }\end{array}$} & \multirow{2}{*}{$\begin{array}{l}\text { ANL } \\
\text { Report } \\
\text { No. }\end{array}$} \\
\hline & $\begin{array}{l}304 \text { SS, } \\
\text { mVISH }\end{array}$ & $\begin{array}{l}\text { Pt. } \\
\text { E) }\end{array}$ & & & & $\begin{array}{r}K_{\max } \text {. } \\
M P a .\end{array}$ & $\underset{1 / 2}{\Delta K, e}$ & $\begin{array}{l}\text { Rate, } \\
10^{-10} \mathrm{~m} \cdot \mathrm{s}^{-1}\end{array}$ & $\begin{array}{r}\mathrm{K}_{\max } \mathrm{d} \\
\mathrm{MPa} .\end{array}$ & $\Delta K$ & $\begin{array}{c}\text { Rate, } \\
10^{-10} \mathrm{~m} \cdot \mathrm{s}^{-1}\end{array}$ & $\begin{array}{l}\text { EPR, } \\
C \mathrm{~cm}^{-2}\end{array}$ & & \\
\hline & $\begin{array}{c}165 \\
130 \\
145 \\
108 \\
95\end{array}$ & $\begin{array}{r}165 \\
130 \\
174 \\
179 \\
95\end{array}$ & $\begin{array}{l}0.90 \\
0.80 \\
0.95 \\
0.95 \\
0.95\end{array}$ & $\begin{array}{l}8 \\
8 \\
8 \\
8 \\
8\end{array}$ & $\begin{array}{l}12 \\
12 \\
12 \\
12 \\
12\end{array}$ & $\begin{array}{l}28.5 \\
25.7 \\
31.4 \\
33.6 \\
35.4\end{array}$ & $\begin{array}{l}2.85 \\
5.12 \\
1.57 \\
1.68 \\
1.77\end{array}$ & $\begin{array}{l}4.50 \\
3.60 \\
0.60 \\
0.80 \\
1.50\end{array}$ & $\begin{array}{l}25.4 \\
25.2 \\
33.4 \\
39.9 \\
40.7\end{array}$ & $\begin{array}{l}2.54 \\
5.03 \\
1.67 \\
2.00 \\
2.04\end{array}$ & $\begin{aligned} & 0.23 \\
& 1.80 \\
& 1.20 \\
& \approx 0 \\
& 0.20\end{aligned}$ & $\begin{array}{l}2 \\
2 \\
2 \\
2 \\
2\end{array}$ & $\begin{array}{l}55 \\
55 \\
55 \\
55 \\
55\end{array}$ & $\begin{array}{l}89-40 \\
89-40 \\
89-40 \\
89-40 \\
89-40\end{array}$ \\
\hline $\begin{array}{l}0.13 \\
0.12\end{array}$ & $\begin{array}{l}195 \\
190\end{array}$ & $\begin{array}{l}200 \\
200\end{array}$ & $\begin{array}{l}0.95 \\
0.95\end{array}$ & $\begin{array}{l}8 \\
8\end{array}$ & $\begin{array}{l}12 \\
12\end{array}$ & $\begin{array}{l}27.7 \\
28.9\end{array}$ & $\begin{array}{l}1.38 \\
1.45\end{array}$ & $\begin{array}{l}0.67 \\
0.90\end{array}$ & $\begin{array}{l}30.4 \\
33.6\end{array}$ & $\begin{array}{l}1.52 \\
1.68\end{array}$ & $\begin{array}{l}2.90 \\
2.90\end{array}$ & $\begin{array}{l}2 \\
2\end{array}$ & $\begin{array}{l}55.56 \\
55.56\end{array}$ & $\begin{array}{l}89-40 \\
890 / 4\end{array}$ \\
\hline $\begin{array}{l}0.16 \\
0.16 \\
0.16 \\
0.16\end{array}$ & $\begin{array}{l}109 \\
109 \\
109 \\
109\end{array}$ & $\begin{array}{l}115 \\
115 \\
115 \\
115\end{array}$ & $\begin{array}{l}0.95 \\
0.80 \\
0.90 \\
0.90\end{array}$ & $\begin{array}{l}8 \\
8 \\
8 \\
8\end{array}$ & $\begin{array}{l}12 \\
12 \\
12 \\
12\end{array}$ & $\begin{array}{l}29.3 \\
32.0 \\
33.7 \\
33.9\end{array}$ & $\begin{array}{l}1.47 \\
1.60 \\
1.68 \\
1.69\end{array}$ & $\begin{array}{r}2.5 \\
i 9.0 \\
6.6 \\
3.6\end{array}$ & $\begin{array}{l}\bar{z} \\
-\end{array}$ & $\begin{array}{l}- \\
- \\
-\end{array}$ & $\begin{array}{l}- \\
- \\
-\end{array}$ & $\begin{array}{l}- \\
- \\
-\end{array}$ & $\begin{array}{l}57 \\
57 \\
57 \\
57\end{array}$ & $\begin{array}{l}91 / 24 \\
91 / 24 \\
91 / 24 \\
91 / 24\end{array}$ \\
\hline $\begin{array}{l}0.16 \\
0.16 \\
0.16 \\
0.16\end{array}$ & $\begin{array}{l}109 \\
109 \\
109 \\
109\end{array}$ & $\begin{array}{l}115 \\
115 \\
115 \\
115\end{array}$ & $\begin{array}{l}0.95 \\
0.80 \\
0.90 \\
0.90\end{array}$ & $\begin{array}{l}8 \\
8 \\
8 \\
8\end{array}$ & $\begin{array}{l}12 \\
12 \\
12 \\
12\end{array}$ & $\begin{array}{l}29.3 \\
30.8 \\
34.5 \\
45.7\end{array}$ & $\begin{array}{l}1.47 \\
1.54 \\
1.72 \\
2.28\end{array}$ & $\begin{array}{l}3.1 \\
34.0 \\
14.0 \\
20.0\end{array}$ & $\begin{array}{l}- \\
- \\
-\end{array}$ & $\begin{array}{l}- \\
- \\
-\end{array}$ & $\begin{array}{l}- \\
- \\
-\end{array}$ & $\begin{array}{l}- \\
- \\
-\end{array}$ & $\begin{array}{l}57 \\
57 \\
57 \\
57\end{array}$ & $\begin{array}{l}91 / 24 \\
91 / 24 \\
91 / 24 \\
91 / 24\end{array}$ \\
\hline $\begin{array}{l}0.17 \\
0.17 \\
0.17\end{array}$ & $\begin{array}{l}119 \\
119 \\
119\end{array}$ & $\begin{array}{l}119 \\
119 \\
119\end{array}$ & $\begin{array}{l}0.95 \\
0.95 \\
0.95\end{array}$ & $\begin{array}{l}8 \\
8 \\
8\end{array}$ & $\begin{array}{l}12 \\
12 \\
12\end{array}$ & $\begin{array}{l}- \\
-\end{array}$ & $\begin{array}{l}- \\
-\end{array}$ & $\begin{array}{l}\overline{-} \\
-\end{array}$ & $\begin{array}{l}27.7 \\
30.9 \\
28.5\end{array}$ & $\begin{array}{l}1.38 \\
1.54 \\
1.42\end{array}$ & $\begin{array}{l}0.05 \\
3.30 \\
0.83\end{array}$ & $\begin{array}{r}0 \\
8 \\
30\end{array}$ & $\begin{array}{l}58 \\
58 \\
58\end{array}$ & $\begin{array}{l}92 / 6 \\
92 / 6 \\
92 / 6\end{array}$ \\
\hline $\begin{array}{l}0.12 \\
0.12 \\
0.12\end{array}$ & $\begin{array}{l}91 \\
91 \\
91\end{array}$ & $\begin{array}{l}66 \\
66 \\
66\end{array}$ & $\begin{array}{l}0.95 \\
0.95 \\
0.95\end{array}$ & $\begin{array}{l}8 \\
8 \\
8\end{array}$ & $\begin{array}{l}12 \\
12 \\
12\end{array}$ & $\begin{array}{l}- \\
-\end{array}$ & $\begin{array}{l}- \\
- \\
-\end{array}$ & $\overline{-}$ & $\begin{array}{l}27.7 \\
31.5 \\
28.6\end{array}$ & $\begin{array}{l}1.38 \\
1.57 \\
1.43\end{array}$ & $\begin{array}{l}0.05 \\
0.43 \\
0.09\end{array}$ & $\begin{array}{r}0 \\
8 \\
30\end{array}$ & $\begin{array}{l}58 \\
58 \\
58\end{array}$ & $\begin{array}{l}92 / 6 \\
92 / 6 \\
92 / 6\end{array}$ \\
\hline
\end{tabular}

aCompact-tension specimens (1TCT) of Type 316NG SS (Hleat No. P91576) and Type 304 SS (Heat No. 30956) with the following heat treatments: solution anneal at $1050^{\circ} \mathrm{C}$ for $0.5 \mathrm{~h}$ plus $650^{\circ} \mathrm{C}$ for $24 \mathrm{~h}$ for the Type $316 \mathrm{NG}$ SS (EPR $=0 \mathrm{C} \cdot \mathrm{cm}^{-2}$ ). and solution anneal at $1050^{\circ} \mathrm{C}$ for $0.5 \mathrm{~h}(\mathrm{EPR}=0 \mathrm{C} \cdot \mathrm{cm}-2$ followed by $700^{\circ} \mathrm{C}$ for $0.25 \mathrm{~h}$ plus $500^{\circ} \mathrm{C}$ for $24 \mathrm{~h}\left(\mathrm{EPR}=2 \mathrm{C} \cdot \mathrm{cm}^{-2}\right.$ ) or $700^{\circ} \mathrm{C}$ for $12 \mathrm{~h}$ (EPR $=20 \mathrm{C} \cdot \mathrm{cm}^{-2}$ ) for the Type $304 \mathrm{SS}$.

bEfluent DO concentration was $200-300$ ppb; feedwater oxygen concentration was approximately a factor of 2 higher to compensate for oxygen depletion by corrosion of the autoclave systems.

CPositive sawtooth waveform.

dStress intensity, $K_{\max }$, values at the end of $a \approx 1000-h$ time period of steady-state crack growth.

$e_{\Delta K}=K_{\max }(1-R)$, where the load ratio $R=K_{\min } / K_{\max }$. 
Table 4. Summary of crack growth results for solution-annealed and sensitized Type 304 SS specimens ${ }^{a}$ in oxygenated ( $\approx 5-8 \mathrm{ppm}$ ) waterb at $289^{\circ} \mathrm{C}$, in which load ratio, frequency, ${ }^{c}$ and stress intensity were varied

\begin{tabular}{|c|c|c|c|c|c|c|c|c|c|c|}
\hline \multirow[b]{2}{*}{$\begin{array}{l}\text { Cond., } \\
\mu \mathrm{S} \cdot \mathrm{cm}^{-1}\end{array}$} & \multirow{2}{*}{$\begin{array}{l}\text { Potential } \\
304 \text { SS, } \\
\text { mV(SHE) }\end{array}$} & \multirow{2}{*}{$\begin{array}{l}\text { Load } \\
\text { Ratio }\end{array}$} & \multirow[b]{2}{*}{$\begin{array}{c}\text { Freq.. } \\
(10-2) \mathrm{Hz}\end{array}$} & \multirow{2}{*}{$\begin{array}{l}\text { Rise } \\
\text { Time, } \\
\text { s }\end{array}$} & \multicolumn{3}{|c|}{ Type 304 SS } & \multirow[b]{2}{*}{$\begin{array}{c}\text { EPR, } \\
\text { C.cm-2 }\end{array}$} & \multirow[b]{2}{*}{$\begin{array}{l}\text { Ref. } \\
\text { No. }\end{array}$} & \multirow{2}{*}{$\begin{array}{l}\text { ANL } \\
\text { Report } \\
\text { No. }\end{array}$} \\
\hline & & & & & $\overline{K_{\max }{ }_{\text {MP.m }}{ }^{1 / 2}}$ & $2^{\Delta \mathrm{K}, \mathrm{e}}$ & $\begin{array}{c}\text { Rate, } \\
(10-10) \mathrm{ms}^{-1}\end{array}$ & & & \\
\hline $\begin{array}{l}<0.2 \\
<0.2 \\
<0.2 \\
<0.2 \\
<0.2\end{array}$ & $\begin{array}{l}\overline{-} \\
\overline{-} \\
\overline{-}\end{array}$ & $\begin{array}{l}1.0 \\
1.0 \\
1.0 \\
1.0 \\
1.0\end{array}$ & $\begin{array}{l}0 \\
0 \\
0 \\
0 \\
0\end{array}$ & $\begin{array}{l}\infty \\
\infty \\
\infty \\
\infty \\
\infty\end{array}$ & $\begin{array}{l}34.0 \\
37.0 \\
38.0 \\
33.0 \\
29.0\end{array}$ & $\begin{array}{l}0 \\
0 \\
0 \\
0 \\
0\end{array}$ & $\begin{array}{l}1.20 \\
2.90 \\
4.50 \\
2.20 \\
1.80\end{array}$ & $\begin{array}{c}1.4 \\
1.4 \\
1.4 \\
1.8 \\
\text { HAZ }\end{array}$ & $\begin{array}{l}\mathbf{5 9} \\
59 \\
59 \\
59 \\
60\end{array}$ & $\begin{array}{l}84-60 \text { III } \\
84-60 \text { III } \\
84-60 \text { III } \\
84-60 \text { III } \\
85-75 \text { I }\end{array}$ \\
\hline $\begin{array}{l}<0.2 \\
<0.2 \\
<0.2\end{array}$ & $\overline{-}$ & $\begin{array}{l}0.95 \\
0.95 \\
0.95\end{array}$ & $\begin{array}{l}8.0 \\
8.0 \\
8.0\end{array}$ & $\begin{array}{l}12 \\
12 \\
12\end{array}$ & $\begin{array}{l}28.0 \\
34.0 \\
35.0\end{array}$ & $\begin{array}{l}1.40 \\
1.70 \\
1.75\end{array}$ & $\begin{array}{l}7.50 \\
10.0 \\
12.0\end{array}$ & $\begin{array}{l}20 \\
20 \\
20\end{array}$ & $\begin{array}{l}61 \\
61 \\
61\end{array}$ & $\begin{array}{l}83-85 \text { II } \\
83-85 \text { II } \\
83-85 \text { II }\end{array}$ \\
\hline $\begin{array}{l}<0.2 \\
<0.2 \\
<0.2 \\
<0.2 \\
<0.2\end{array}$ & $\begin{array}{l}\overline{-} \\
\overline{-} \\
-\end{array}$ & $\begin{array}{l}0.95 \\
0.95 \\
0.95 \\
0.95 \\
0.95\end{array}$ & $\begin{array}{l}0.8 \\
0.8 \\
0.8 \\
0.8 \\
0.8\end{array}$ & $\begin{array}{l}124 \\
124 \\
124 \\
124 \\
124\end{array}$ & $\begin{array}{l}34.0 \\
38.0 \\
50.0 \\
61.0 \\
64.0\end{array}$ & $\begin{array}{l}1.70 \\
1.90 \\
2.50 \\
3.04 \\
3.20\end{array}$ & $\begin{array}{l}1.20 \\
1.50 \\
4.70 \\
11.0 \\
17.0\end{array}$ & $\begin{array}{l}20 \\
20 \\
20 \\
20 \\
20\end{array}$ & $\begin{array}{l}61 \\
61 \\
61 \\
61 \\
61\end{array}$ & $\begin{array}{l}83-85 \text { II } \\
83-85 \text { II } \\
83-85 \text { II } \\
83-85 \text { II } \\
83-85 \text { II }\end{array}$ \\
\hline $\begin{array}{l}<0.2 \\
<0.2 \\
<0.2 \\
<0.2\end{array}$ & $\begin{array}{l}- \\
-\end{array}$ & $\begin{array}{l}0.95 \\
0.95 \\
0.95 \\
0.95\end{array}$ & $\begin{array}{l}0.08 \\
0.08 \\
0.08 \\
0.08\end{array}$ & $\begin{array}{l}1250 \\
1250 \\
1250 \\
1250\end{array}$ & $\begin{array}{l}28.0 \\
67.0 \\
70.0 \\
72.0\end{array}$ & $\begin{array}{l}1.40 \\
3.35 \\
3.50 \\
3.60\end{array}$ & $\begin{array}{l}1.20 \\
19.0 \\
32.0 \\
33.0\end{array}$ & $\begin{array}{l}20 \\
20 \\
20 \\
20\end{array}$ & $\begin{array}{l}61 \\
61 \\
61 \\
61\end{array}$ & $\begin{array}{l}83-85 \text { II } \\
83-85 \text { II } \\
83-85 \\
83-85\end{array}$ \\
\hline $\begin{array}{l}<0.2 \\
<0.2 \\
<0.2 \\
<0.2 \\
<0.2\end{array}$ & $\begin{array}{l}- \\
\overline{-} \\
z\end{array}$ & $\begin{array}{l}0.95 \\
0.95 \\
0.95 \\
0.95 \\
0.95\end{array}$ & $\begin{array}{l}0.2 \\
0.2 \\
0.2 \\
0.2 \\
0.2\end{array}$ & $\begin{array}{l}495 \\
495 \\
495 \\
495 \\
495\end{array}$ & $\begin{array}{l}36.0 \\
37.0 \\
39.0 \\
40.0 \\
28.0\end{array}$ & $\begin{array}{l}1.70 \\
1.85 \\
1.95 \\
2.00 \\
1.40\end{array}$ & $\begin{array}{l}1.70 \\
1.50 \\
2.00 \\
3.10 \\
2.20\end{array}$ & $\begin{array}{r}1.4 \\
1.4 \\
1.4 \\
1.4 \\
\mathrm{HAZ}\end{array}$ & $\begin{array}{l}\mathbf{5 9} \\
59 \\
59 \\
59 \\
60\end{array}$ & $\begin{array}{l}84-60 \text { III } \\
84-60 \text { III } \\
84-60 \text { III } \\
84-60 \text { III } \\
85-75 \text { I }\end{array}$ \\
\hline $\begin{array}{l}<0.2 \\
<0.2 \\
<0.2\end{array}$ & $\overline{-}$ & $\begin{array}{l}0.94 \\
0.94 \\
0.94\end{array}$ & $\begin{array}{l}10.0 \\
10.0 \\
10.0\end{array}$ & $\begin{array}{l}\mathbf{5} \\
\mathbf{5} \\
\mathbf{5}\end{array}$ & $\begin{array}{l}31.0 \\
32.0 \\
30.0\end{array}$ & $\begin{array}{l}1.86 \\
1.92 \\
1.80\end{array}$ & $\begin{array}{l}3.10 \\
1.90 \\
2.10\end{array}$ & $\begin{array}{l}1.4 \\
1.4 \\
1.8\end{array}$ & $\begin{array}{l}59 \\
59 \\
59\end{array}$ & $\begin{array}{l}84-60 \text { III } \\
84-60 \text { III } \\
84-60 \text { III }\end{array}$ \\
\hline $\begin{array}{l}<0.2 \\
<0.2\end{array}$ & $\overline{-}$ & $\begin{array}{l}0.90 \\
0.80\end{array}$ & $\begin{array}{l}0.2 \\
0.2\end{array}$ & $\begin{array}{l}495 \\
495\end{array}$ & $\begin{array}{l}28.0 \\
29.0\end{array}$ & $\begin{array}{l}2.80 \\
5.80\end{array}$ & $\begin{array}{l}1.30 \\
6.60\end{array}$ & $\begin{array}{l}\text { HAZ } \\
\text { HAZ }\end{array}$ & $\begin{array}{l}60 \\
60\end{array}$ & $\begin{array}{l}85-75 \text { I } \\
85-75\end{array}$ \\
\hline $\begin{array}{l}<0.2 \\
<0.2 \\
<0.2 \\
<0.2\end{array}$ & $\begin{array}{l}- \\
- \\
-\end{array}$ & $\begin{array}{l}0.80 \\
0.80 \\
0.79 \\
0.79\end{array}$ & $\begin{array}{l}0.2 \\
0.2 \\
0.2 \\
0.2\end{array}$ & $\begin{array}{l}495 \\
495 \\
495 \\
495\end{array}$ & $\begin{array}{l}31.0 \\
32.0 \\
32.0 \\
36.0\end{array}$ & $\begin{array}{l}6.20 \\
6.39 \\
6.70 \\
7.55\end{array}$ & $\begin{array}{l}4.40 \\
7.40 \\
5.50 \\
5.40\end{array}$ & $\begin{array}{l}1.4 \\
1.4 \\
1.4 \\
1.4\end{array}$ & $\begin{array}{l}59 \\
59 \\
59 \\
59\end{array}$ & $\begin{array}{l}84-60 \text { III } \\
84-60 \text { III } \\
84-60 \text { III } \\
84-60 \text { III }\end{array}$ \\
\hline $\begin{array}{l}<0.2 \\
<0.2\end{array}$ & $\overline{-}$ & $\begin{array}{l}0.70 \\
0.70\end{array}$ & $\begin{array}{l}0.2 \\
0.2\end{array}$ & $\begin{array}{l}495 \\
495\end{array}$ & $\begin{array}{l}31.0 \\
33.0\end{array}$ & $\begin{array}{l}9.30 \\
9.90\end{array}$ & $\begin{array}{l}3.40 \\
5.90\end{array}$ & $\begin{array}{l}1.4 \\
1.4\end{array}$ & $\begin{array}{l}59 \\
59\end{array}$ & $\begin{array}{l}84-60 \text { III } \\
84-60 \text { III }\end{array}$ \\
\hline $\begin{array}{l}<0.2 \\
<0.2\end{array}$ & $\overline{-}$ & $\begin{array}{l}0.60 \\
0.60\end{array}$ & $\begin{array}{l}0.2 \\
0.2\end{array}$ & $\begin{array}{l}495 \\
495\end{array}$ & $\begin{array}{l}29.0 \\
33.0\end{array}$ & $\begin{array}{l}11.60 \\
13.20\end{array}$ & $\begin{array}{l}56.0 \\
6.60\end{array}$ & $\begin{array}{l}1.8 \\
1.4\end{array}$ & $\begin{array}{l}59 \\
59\end{array}$ & $\begin{array}{l}84-60 \text { III } \\
84-60 \text { III }\end{array}$ \\
\hline $\begin{array}{l}<0.2 \\
<0.2 \\
<0.2 \\
<0.2\end{array}$ & $\begin{array}{l}\bar{z} \\
\overline{-}\end{array}$ & $\begin{array}{l}0.50 \\
0.50 \\
0.50 \\
0.50\end{array}$ & $\begin{array}{l}0.1 \\
0.2 \\
0.2 \\
0.2\end{array}$ & $\begin{array}{l}995 \\
495 \\
495 \\
495\end{array}$ & $\begin{array}{l}32.0 \\
31.0 \\
33.0 \\
32.0\end{array}$ & $\begin{array}{l}16.00 \\
15.50 \\
16.50 \\
16.00\end{array}$ & $\begin{array}{l}2.60 \\
8.90 \\
34.0 \\
28.0\end{array}$ & $\begin{array}{l}1.4 \\
1.4 \\
1.4 \\
1.8\end{array}$ & $\begin{array}{l}\mathbf{5 9} \\
59 \\
59 \\
59\end{array}$ & $\begin{array}{l}84-60 \text { III } \\
84-60 \text { II } \\
84-60 \text { III } \\
84-60 \text { III }\end{array}$ \\
\hline $\begin{array}{l}0.12 \\
0.12 \\
0.12\end{array}$ & $\begin{array}{l}206 \\
206 \\
206\end{array}$ & $\begin{array}{l}0.95 \\
0.95 \\
0.95\end{array}$ & $\begin{array}{l}8.0 \\
8.0 \\
8.0\end{array}$ & $\begin{array}{l}12 \\
12 \\
12\end{array}$ & $\begin{array}{l}27.6 \\
29.2 \\
28.2\end{array}$ & $\begin{array}{l}1.38 \\
1.46 \\
1.41\end{array}$ & $\begin{array}{l}0.05 \\
9.2 \\
1.0\end{array}$ & $\begin{array}{r}0 \\
8 \\
30\end{array}$ & $\begin{array}{l}58 \\
58 \\
58\end{array}$ & $\begin{array}{l}92 / 6 \\
92 / 6 \\
92 / 6\end{array}$ \\
\hline
\end{tabular}

aCompact-tenston specimens (1TCT) of Type 304 SS with the following heat treatments: Heat No. 10285 , solution anneal at $1050^{\circ} \mathrm{C}$ for $0.5 \mathrm{~h}$ plus $700^{\circ} \mathrm{C} / 10 \mathrm{~min}$ and $450^{\circ} \mathrm{C} / 146 \mathrm{~h}$ or $450^{\circ} \mathrm{C} / 250 \mathrm{~h}$ $\left(E P R=1.4 \mathrm{C} \cdot \mathrm{cm}^{-2}\right)$, or $500^{\circ} \mathrm{C} / 24 \mathrm{~h}\left(E P R=1.8 \mathrm{C} \cdot \mathrm{cm}^{-2}\right)$. Heat No. 30956 solution anneal at $1050^{\circ} \mathrm{C}$ for $0.5 \mathrm{~h}$ and quenched (EPR $=0 \mathrm{C} \cdot \mathrm{cm}^{-2}$ ) followed by $700^{\circ} \mathrm{C}$ for $0.67 \mathrm{~h}\left(\mathrm{EPR}=8 \mathrm{C} \cdot \mathrm{cm}^{-2}\right), 700^{\circ} \mathrm{C}$ for $12 \mathrm{~h}\left(E P R=20 \mathrm{C} \cdot \mathrm{cm}^{-2}\right)$, or $700^{\circ} \mathrm{C}$ for $24 \mathrm{~h}\left(E P R=30 \mathrm{C} \cdot \mathrm{cm}^{-2}\right)$. Heat affected zone (HAZ) specimen was fabricated from a weld overiay applied to a 12-in. diam pipe.

bEffluent DO concentration was $5-8$ ppm.

CPositive sawtooth waveform was used.

dStress intensity, $K_{\max }$, values at the end of $a \approx 500-1000-h$ time period of steady-state crack growth.

$e_{\Delta K}=K_{\max }(1-R)$, where the load ratio $R=K_{\min } / K_{\max }$. 
Table 5. Summary of crack growth results for Type 316NG and sensitized and solution-annealed Type 304 SS specimens in axygenated $(\approx 200 \mathrm{ppb})$ water ${ }^{b}$ with ionic impurities at $289^{\circ} \mathrm{C}$, in which load ratioc and stress intensity were varied

\begin{tabular}{|c|c|c|c|c|c|c|c|c|c|c|c|c|c|c|c|c|c|}
\hline \multicolumn{4}{|c|}{ Water Chemistry } & \multicolumn{2}{|c|}{ Potentials } & \multirow{2}{*}{$\begin{array}{l}\text { Load } \\
\text { Ratio }\end{array}$} & \multirow[b]{2}{*}{$\begin{array}{r}\text { Freq.. } \\
10^{-2} \mathrm{~Hz}\end{array}$} & \multirow{2}{*}{$\begin{array}{l}\text { Rise } \\
\text { Time. } \\
\text { s }\end{array}$} & \multicolumn{3}{|c|}{ Type 316NG SS } & \multicolumn{4}{|c|}{ Type 304 SS } & \multirow[b]{2}{*}{$\begin{array}{l}\text { Ref. } \\
\text { No. }\end{array}$} & \multirow{2}{*}{$\begin{array}{c}\text { ANL } \\
\text { Report } \\
\text { No. }\end{array}$} \\
\hline $\begin{array}{l}\text { Cond. } \\
\mu S \mathrm{~cm}^{-1}\end{array}$ & $\begin{array}{l}\mathrm{SO}_{4}^{2-} \\
\mathrm{ppb}\end{array}$ & $\begin{array}{l}\mathrm{CrO}_{4}^{2-} . \\
\mathrm{Ppb}\end{array}$ & $\begin{array}{l}\text { Other. } \\
\text { ppb }\end{array}$ & $\begin{array}{l}304 \text { SS. } \\
\text { mVISH }\end{array}$ & $\begin{array}{l}\text { Pt. } \\
\text { E) }\end{array}$ & & & & $\begin{array}{l}K_{\max }^{d} \cdot \\
M P a \cdot m\end{array}$ & $\underset{1 / 2}{\Delta K e}$ & $\begin{array}{c}\text { Rate. } \\
10^{-10} \mathrm{~m} \cdot \mathrm{s}^{-1}\end{array}$ & $\begin{array}{l}K_{\max }^{d} \cdot \\
M P a \cdot n\end{array}$ & $\begin{array}{l}\Delta K, e \\
n^{1 / 2}\end{array}$ & $\begin{array}{c}\text { Rate.g } \\
10^{-10} \mathrm{~m} \cdot \mathrm{s}^{-1}\end{array}$ & $\begin{array}{c}\text { EPR. } \\
\text { C. } \mathrm{cm}^{-2}\end{array}$ & & \\
\hline $\begin{array}{l}0.92 \\
1.04 \\
0.92 \\
1.04 \\
0.92 \\
1.04\end{array}$ & $\begin{array}{l}100 \\
100 \\
100 \\
100 \\
100 \\
100\end{array}$ & $\begin{array}{l}- \\
\overline{-} \\
z \\
-\end{array}$ & $\begin{array}{l}- \\
- \\
z \\
-\end{array}$ & $\begin{array}{l}- \\
- \\
z \\
z\end{array}$ & $\begin{array}{l}- \\
\overline{-} \\
\overline{-} \\
-\end{array}$ & $\begin{array}{l}0.95 \\
0.95 \\
0.95 \\
0.95 \\
0.95 \\
0.95\end{array}$ & $\begin{array}{l}8 \\
8 \\
8 \\
8 \\
8 \\
8\end{array}$ & $\begin{array}{l}12 \\
12 \\
12 \\
12 \\
12 \\
12\end{array}$ & $\begin{array}{l}- \\
- \\
- \\
-\end{array}$ & $\begin{array}{l}- \\
\overline{-} \\
\overline{-} \\
-\end{array}$ & $\begin{array}{l}- \\
\overline{-} \\
- \\
- \\
-\end{array}$ & $\begin{array}{l}42.1 \\
49.8 \\
53.2 \\
63.4 \\
42.0 \\
42.2\end{array}$ & $\begin{array}{l}2.10 \\
2.49 \\
2.66 \\
3.17 \\
2.10 \\
2.11\end{array}$ & $\begin{array}{c}6.10 \\
6.90 \\
6.40 \\
16.0 \\
1.60 \\
1.00\end{array}$ & $\begin{array}{r}0 \\
0 \\
2 \\
2 \\
20 \\
20\end{array}$ & $\begin{array}{l}49 \\
49 \\
49 \\
49 \\
49 \\
49\end{array}$ & $\begin{array}{l}83-85 \text { IV } \\
83-85 \text { IV } \\
83-85 \text { IV } \\
83-85 \text { IV } \\
83-85 \text { IV } \\
83-85 \text { IV }\end{array}$ \\
\hline $\begin{array}{l}0.88 \\
0.88 \\
0.88\end{array}$ & $\begin{array}{l}100 \\
100 \\
100\end{array}$ & $\begin{array}{l}- \\
-\end{array}$ & $\overline{-}$ & $\begin{array}{r}80 \\
120 \\
120\end{array}$ & $\begin{array}{l}180 \\
215 \\
190\end{array}$ & $\begin{array}{l}0.95 \\
0.95 \\
0.95\end{array}$ & $\begin{array}{l}8 \\
8 \\
8\end{array}$ & $\begin{array}{l}12 \\
12 \\
12\end{array}$ & $\begin{array}{l}- \\
-\end{array}$ & $\begin{array}{l}- \\
-\end{array}$ & $\overline{-}$ & $\begin{array}{l}26.5 \\
28.1 \\
32.3\end{array}$ & $\begin{array}{l}1.33 \\
1.41 \\
1.62\end{array}$ & $\begin{array}{l}2.80 \\
4.20 \\
4.20\end{array}$ & $\begin{array}{l}0 \\
0 \\
0\end{array}$ & $\begin{array}{l}60 \\
60 \\
60\end{array}$ & $\begin{array}{l}85-75 \\
85-75 \\
85-75\end{array}$ \\
\hline $\begin{array}{l}0.88 \\
0.88 \\
0.88\end{array}$ & $\begin{array}{l}100 \\
100 \\
100\end{array}$ & $\begin{array}{l}- \\
-\end{array}$ & $\overline{-}$ & $\begin{array}{r}80 \\
120 \\
120\end{array}$ & $\begin{array}{l}180 \\
215 \\
190\end{array}$ & $\begin{array}{l}0.95 \\
0.95 \\
0.95\end{array}$ & $\begin{array}{l}8 \\
8 \\
8\end{array}$ & $\begin{array}{l}12 \\
12 \\
12\end{array}$ & $\begin{array}{l}- \\
-\end{array}$ & $\begin{array}{l}- \\
-\end{array}$ & $\begin{array}{l}- \\
-\end{array}$ & $\begin{array}{l}27.0 \\
30.0 \\
32.4\end{array}$ & $\begin{array}{l}1.35 \\
1.50 \\
1.62\end{array}$ & $\begin{array}{l}5.00 \\
3.40 \\
2.90\end{array}$ & $\begin{array}{l}2 \\
2 \\
2\end{array}$ & $\begin{array}{l}60 \\
60 \\
60\end{array}$ & $\begin{array}{l}85-75 \\
85-75 \\
85-75\end{array}$ \\
\hline $\begin{array}{l}0.88 \\
0.88 \\
0.88\end{array}$ & $\begin{array}{l}100 \\
100 \\
100\end{array}$ & $\begin{array}{l}- \\
-\end{array}$ & $\overline{-}$ & $\begin{array}{r}80 \\
120 \\
120\end{array}$ & $\begin{array}{l}180 \\
215 \\
190\end{array}$ & $\begin{array}{l}0.95 \\
0.95 \\
0.95\end{array}$ & $\begin{array}{l}8 \\
8 \\
8\end{array}$ & $\begin{array}{l}12 \\
12 \\
12\end{array}$ & $\begin{array}{l}- \\
-\end{array}$ & $\begin{array}{l}- \\
-\end{array}$ & $\begin{array}{l}- \\
-\end{array}$ & $\begin{array}{l}27.1 \\
29.3 \\
31.4\end{array}$ & $\begin{array}{l}1.36 \\
1.46 \\
1.57\end{array}$ & $\begin{array}{l}4.20 \\
3.40 \\
2.60\end{array}$ & $\begin{array}{l}20 \\
20 \\
20\end{array}$ & $\begin{array}{l}60 \\
60 \\
60\end{array}$ & $\begin{array}{l}85-75 \\
85-75 \\
85-75\end{array}$ \\
\hline $\begin{array}{l}0.86 \\
0.88 \\
0.29\end{array}$ & $\begin{array}{r}100 \\
100 \\
30\end{array}$ & $\begin{array}{l}- \\
-\end{array}$ & $\begin{array}{l}- \\
-\end{array}$ & $\begin{array}{l}120 \\
120 \\
145\end{array}$ & $\begin{array}{l}225 \\
190 \\
230\end{array}$ & $\begin{array}{l}0.95 \\
0.95 \\
0.95\end{array}$ & $\begin{array}{l}8 \\
8 \\
8\end{array}$ & $\begin{array}{l}12 \\
12 \\
12\end{array}$ & $\begin{array}{l}29.7 \\
32.2 \\
36.3\end{array}$ & $\begin{array}{l}1.49 \\
1.61 \\
1.82\end{array}$ & $\begin{array}{l}1.70 \\
1.90 \\
1.30\end{array}$ & $\begin{array}{l}31.6 \\
33.8 \\
38.9\end{array}$ & $\begin{array}{l}1.58 \\
1.69 \\
1.95\end{array}$ & $\begin{array}{l}2.30 \\
3.20 \\
0.60\end{array}$ & $\begin{array}{l}2 \\
2 \\
2\end{array}$ & $\begin{array}{l}51 \\
51 \\
51\end{array}$ & $\begin{array}{l}87-37 \\
87-37 \\
87-37\end{array}$ \\
\hline $\begin{array}{l}0.35 \\
0.93 \\
0.93\end{array}$ & $\begin{array}{r}30 \\
100 \\
100\end{array}$ & I & $\overline{-}$ & $\begin{array}{r}85 \\
65 \\
115\end{array}$ & $\begin{array}{r}90 \\
65 \\
130\end{array}$ & $\begin{array}{l}0.95 \\
0.95 \\
0.95\end{array}$ & $\begin{array}{l}8 \\
8 \\
8\end{array}$ & $\begin{array}{l}12 \\
12 \\
12\end{array}$ & $\begin{array}{c}28.0 \\
- \\
-\end{array}$ & $\begin{array}{l}1.40 \\
-\end{array}$ & $\begin{aligned}= & 0 \\
& -\end{aligned}$ & $\begin{array}{l}32.1 \\
34.3 \\
42.6\end{array}$ & $\begin{array}{l}1.61 \\
1.72 \\
2.14\end{array}$ & $\begin{array}{l}2.20 \\
4.40 \\
8.00\end{array}$ & $\begin{array}{l}2 \\
2 \\
2\end{array}$ & $\begin{array}{l}\mathbf{5 2} \\
\mathbf{5 2} \\
\mathbf{5 2}\end{array}$ & $\begin{array}{l}87-41 \\
87-41 \\
87-41\end{array}$ \\
\hline 0.80 & 100 & - & - & 190 & 220 & 0.95 & 8 & 12 & 34.4 & 1.72 & 0.79 & - & - & - & - & 53 & $88-32$ \\
\hline 0.77 & - & - & $100 \mathrm{NO}_{3}^{-}$ & 200 & 215 & 0.95 & 8 & 12 & 33.7 & 1.68 & 0.84 & - & - & - & - & 53 & $88-32$ \\
\hline $\begin{array}{l}0.53 \\
0.91 \\
0.51 \\
0.28 \\
0.54 \\
0.94 \\
0.21 \\
0.51\end{array}$ & $\begin{array}{r}50 \\
100 \\
50 \\
25 \\
50 \\
100 \\
50\end{array}$ & $\begin{array}{l}- \\
- \\
- \\
- \\
\overline{25} \\
-\end{array}$ & $\begin{array}{l}- \\
- \\
- \\
- \\
- \\
-\end{array}$ & $\begin{array}{l}180 \\
110 \\
162 \\
145 \\
117 \\
138 \\
100 \\
162\end{array}$ & $\begin{array}{l}180 \\
194 \\
218 \\
199 \\
194 \\
209 \\
179 \\
218\end{array}$ & $\begin{array}{l}0.95 \\
0.95 \\
0.95 \\
0.95 \\
0.95 \\
0.95 \\
0.95 \\
0.92\end{array}$ & $\begin{array}{l}8 \\
8 \\
8 \\
8 \\
8 \\
8 \\
8 \\
8\end{array}$ & $\begin{array}{l}12 \\
12 \\
12 \\
12 \\
12 \\
12 \\
12 \\
12\end{array}$ & \begin{tabular}{l|}
30.0 \\
28.6 \\
28.8 \\
31.0 \\
32.1 \\
33.2 \\
34.6 \\
30.5
\end{tabular} & $\begin{array}{l}1.50 \\
1.43 \\
1.44 \\
1.55 \\
1.61 \\
1.66 \\
1.73 \\
2.44\end{array}$ & $\begin{array}{l}0.86 \\
1.90 \\
1.50 \\
1.00 \\
1.30 \\
1.60 \\
1.70 \\
4.30\end{array}$ & $\begin{array}{l}29.9 \\
30.0 \\
30.2 \\
32.6 \\
35.4 \\
39.7 \\
40.6 \\
32.0\end{array}$ & $\begin{array}{l}1.50 \\
1.50 \\
1.51 \\
1.63 \\
1.77 \\
1.99 \\
2.03 \\
2.56\end{array}$ & $\begin{array}{l}3.10 \\
2.30 \\
1.10 \\
1.10 \\
2.80 \\
2.30 \\
0.20 \\
4.50\end{array}$ & $\begin{array}{l}2 \\
2 \\
2 \\
2 \\
2 \\
2 \\
2 \\
2\end{array}$ & $\begin{array}{l}\mathbf{5 5} \\
\mathbf{5 5} \\
\mathbf{5 5} \\
\mathbf{5 5} \\
\mathbf{5 5} \\
\mathbf{5 5} \\
\mathbf{5 5} \\
\mathbf{5 5}\end{array}$ & $\begin{array}{l}89-40 \\
89-40 \\
89-40 \\
89-40 \\
89-40 \\
89-40 \\
89-40 \\
89-40\end{array}$ \\
\hline
\end{tabular}


Table 5. Continued

\begin{tabular}{|c|c|c|c|c|c|c|c|c|c|c|c|c|c|c|c|c|c|}
\hline \multicolumn{4}{|c|}{ Water Chemistry } & \multicolumn{2}{|c|}{ Potentials } & \multirow{2}{*}{$\begin{array}{l}\text { Load } \\
\text { Ratio }\end{array}$} & \multirow{2}{*}{$\begin{array}{r}\text { Freq.. } \\
10^{-2} \mathrm{~Hz}\end{array}$} & \multirow{2}{*}{$\begin{array}{c}\text { Rise } \\
\text { Time. } \\
\text { s }\end{array}$} & \multicolumn{3}{|c|}{ Type 316NG SS } & \multicolumn{4}{|c|}{ Type 304 SS } & \multirow[b]{2}{*}{$\begin{array}{l}\text { Ref. } \\
\text { No. }\end{array}$} & \multirow{2}{*}{$\begin{array}{c}\text { ANL } \\
\text { Report } \\
\text { No. }\end{array}$} \\
\hline $\begin{array}{l}\text { Cond. } \\
\mu S \mathrm{~cm}^{-1}\end{array}$ & $\begin{array}{l}\mathrm{SO}_{4}^{2-} \\
\mathrm{ppb}\end{array}$ & $\begin{array}{l}\mathrm{CrO}_{4}^{2-} . \\
\mathrm{ppb}\end{array}$ & $\begin{array}{c}\text { Other. } \\
\text { ppb }\end{array}$ & $\begin{array}{r}304 \text { SS, } \\
\text { mV/S }\end{array}$ & Pt. & & & & $\begin{array}{l}K_{\max }^{d} . \\
M P a \cdot n\end{array}$ & $\mathbf{m}^{\Delta K}$ & $\begin{array}{c}\text { Rate. } \\
10^{-10} \mathrm{~m} \cdot \mathrm{s}^{-1}\end{array}$ & $\begin{array}{l}K_{\max }{ }^{d} \cdot \\
M P a \cdot r\end{array}$ & $\mathrm{~m}^{\Delta K_{\mathbf{e}} \mathrm{e}}$ & $\begin{array}{c}\text { Rate. } \\
10^{-10} \mathrm{~m} \cdot \mathrm{s}^{1}\end{array}$ & $\begin{array}{c}\text { EPR. } \\
\text { C } \mathrm{cm}^{-2}\end{array}$ & & \\
\hline $\begin{array}{l}0.48 \\
3.0\end{array}$ & $\overline{-}$ & $=1$ & $\begin{array}{l}100 \text { Propionic } \\
1000 \text { Propionic }\end{array}$ & $\begin{array}{l}190 \\
206\end{array}$ & $\begin{array}{l}190 \\
220\end{array}$ & $\begin{array}{l}0.95 \\
0.95\end{array}$ & $\begin{array}{l}8 \\
8\end{array}$ & $\begin{array}{l}12 \\
12\end{array}$ & $\begin{array}{l}28.0 \\
28.3\end{array}$ & $\begin{array}{l}1.40 \\
1.41\end{array}$ & $\begin{array}{l}0.64 \\
0.67\end{array}$ & $\begin{array}{l}31.4 \\
31.6\end{array}$ & $\begin{array}{l}1.57 \\
1.58\end{array}$ & $\begin{array}{l}2.90 \\
0.20\end{array}$ & \multicolumn{2}{|c|}{$\begin{array}{ll}2 & 55.56 \\
2 & 55.56\end{array}$} & $\begin{array}{l}89-40 \& \\
90 / 4\end{array}$ \\
\hline 2.5 & - & -1 & 1000 Butyric & 170 & 180 & 0.95 & 8 & 12 & 29.2 & 1.46 & 0.64 & 33.6 & 1.68 & $=0$ & \multicolumn{2}{|c|}{255.56} & $\Downarrow$ \\
\hline 0.38 & - & - & 100 Butyric & 180 & 190 & 0.95 & 8 & 12 & 30.2 & 1.51 & 1.20 & 34.8 & 1.74 & 1.90 & \multicolumn{2}{|c|}{255.56} & $\Downarrow$ \\
\hline 3.7 & 100 & -1 & 1000 Butyric & 190 & 190 & 0.95 & 8 & 12 & 30.5 & 1.52 & 1.30 & 37.0 & 1.85 & 7.40 & \multicolumn{2}{|c|}{255.56} & $\Downarrow$ \\
\hline 3.0 & - & -1 & 1000 Butyric & 190 & 230 & 0.95 & 8 & 12 & 30.6 & 1.53 & 0.09 & 37.0 & 1.85 & $\approx 0$ & \multicolumn{2}{|c|}{255.56} & $\Downarrow$ \\
\hline 3.2 & - & -1 & $\begin{array}{l}1000 \text { Butyric + } \\
100 \mathrm{Cl}^{-}\end{array}$ & 200 & 210 & 0.95 & 8 & 12 & 34.5 & 1.72 & 3.70 & 39.8 & 1.97 & 1.50 & \multicolumn{2}{|c|}{255.56} & $\Downarrow$ \\
\hline 3.1 & - & -1 & 1000 Butyric & 175 & 220 & 0.95 & 8 & 12 & 35.8 & 1.79 & 4.00 & 39.8 & 1.97 & $=0$ & \multicolumn{2}{|c|}{255.56} & $\downarrow$ \\
\hline $\begin{array}{l}0.9 \\
0.9 \\
0.9\end{array}$ & $\begin{array}{l}100 \\
100 \\
100\end{array}$ & $\begin{array}{l}- \\
-\end{array}$ & $\begin{array}{l}- \\
-\end{array}$ & $\begin{array}{r}136 \\
106 \\
81\end{array}$ & $\begin{array}{r}120 \\
66 \\
41\end{array}$ & $\begin{array}{l}0.95 \\
0.25^{\prime} \\
0.95\end{array}$ & $\begin{array}{r}8 \\
10 \\
8\end{array}$ & $\begin{array}{r}12 \\
5 \\
12\end{array}$ & $\begin{array}{l}21.4 \\
19.0 \\
21.6\end{array}$ & $\begin{array}{r}1.07 \\
14.20 \\
1.08\end{array}$ & $\begin{array}{l}0.01^{h} \\
2.60^{h} \\
0.93^{h}\end{array}$ & $\begin{array}{l}- \\
-\end{array}$ & - & $\begin{array}{l}- \\
-\end{array}$ & $\begin{array}{l}- \\
-\end{array}$ & $\begin{array}{l}56 \\
56 \\
56\end{array}$ & $\begin{array}{l}90 / 4 \\
90 / 4 \\
90 / 4\end{array}$ \\
\hline 1.1 & 100 & - & - & 134 & 95 & 1.00 & 0 & $\infty$ & 21.7 & 0.00 & $0.44^{h}$ & - & & - & - & 56 & $90 / 4$ \\
\hline $\begin{array}{l}0.30 \\
0.30 \\
0.30\end{array}$ & $\begin{array}{l}- \\
-\end{array}$ & $\begin{array}{l}50 \\
50 \\
50\end{array}$ & $\begin{array}{l}- \\
-\end{array}$ & $\begin{array}{l}67 \\
67 \\
67\end{array}$ & $\begin{array}{l}58 \\
58 \\
58\end{array}$ & $\begin{array}{l}0.95 \\
0.95 \\
0.95\end{array}$ & $\begin{array}{l}8 \\
8 \\
8\end{array}$ & $\begin{array}{l}12 \\
12 \\
12\end{array}$ & $\overline{-}$ & $\begin{array}{l}- \\
-\end{array}$ & $\begin{array}{l}- \\
-\end{array}$ & $\begin{array}{l}27.7 \\
31.2 \\
28.5\end{array}$ & $\begin{array}{l}1.38 \\
1.56 \\
1.42\end{array}$ & $\begin{array}{l}0.05 \\
0.43 \\
0.09\end{array}$ & $\begin{array}{r}0 \\
8 \\
30\end{array}$ & $\begin{array}{l}58 \\
58 \\
58\end{array}$ & $\begin{array}{l}92 / 6 \\
92 / 6 \\
92 / 6\end{array}$ \\
\hline $\begin{array}{l}0.82 \\
0.82 \\
0.82\end{array}$ & $\begin{array}{l}- \\
-\end{array}$ & $\begin{array}{l}200 \\
200 \\
200\end{array}$ & $\overline{-}$ & $\begin{array}{l}80 \\
80 \\
80\end{array}$ & $\begin{array}{l}80 \\
80 \\
80\end{array}$ & $\begin{array}{l}0.95 \\
0.95 \\
0.95\end{array}$ & $\begin{array}{l}8 \\
8 \\
8\end{array}$ & $\begin{array}{l}12 \\
12 \\
12\end{array}$ & $\overline{-}$ & $\overline{-}$ & $\begin{array}{l}- \\
-\end{array}$ & $\begin{array}{l}27.7 \\
31.8 \\
28.7\end{array}$ & $\begin{array}{l}1.38 \\
1.59 \\
1.43\end{array}$ & $\begin{array}{l}0.05 \\
0.43 \\
0.09\end{array}$ & $\begin{array}{r}\mathbf{0} \\
\mathbf{8} \\
\mathbf{3 0}\end{array}$ & $\begin{array}{l}\mathbf{5 8} \\
\mathbf{5 8} \\
\mathbf{5 8}\end{array}$ & $\begin{array}{l}92 / 6 \\
92 / 6 \\
92 / 6\end{array}$ \\
\hline $\begin{array}{l}0.31 \\
0.31 \\
0.31\end{array}$ & $\begin{array}{l}- \\
-\end{array}$ & $\begin{array}{l}50 \\
50 \\
50\end{array}$ & $\begin{array}{l}- \\
-\end{array}$ & $\begin{array}{l}58 \\
58 \\
58\end{array}$ & $\begin{array}{l}51 \\
51 \\
51\end{array}$ & $\begin{array}{l}0.95 \\
0.95 \\
0.95\end{array}$ & $\begin{array}{l}8 \\
8 \\
8\end{array}$ & $\begin{array}{l}12 \\
12 \\
12\end{array}$ & $\overline{-}$ & - & $\begin{array}{l}- \\
\overline{-}\end{array}$ & $\begin{array}{l}27.8 \\
31.8 \\
28.7\end{array}$ & $\begin{array}{l}1.39 \\
1.59 \\
1.43\end{array}$ & $\begin{array}{l}0.05 \\
0.43 \\
0.09\end{array}$ & $\begin{array}{r}\mathbf{0} \\
\mathbf{8} \\
\mathbf{3 0}\end{array}$ & $\begin{array}{l}58 \\
58 \\
58\end{array}$ & $\begin{array}{l}92 / 6 \\
92 / 6 \\
92 / 6\end{array}$ \\
\hline $\begin{array}{l}0.46 \\
0.46 \\
0.46\end{array}$ & $\begin{array}{l}25 \\
25 \\
25\end{array}$ & $\begin{array}{l}50 \\
50 \\
50\end{array}$ & $\begin{array}{l}- \\
-\end{array}$ & $\begin{array}{l}91 \\
91 \\
91\end{array}$ & $\begin{array}{l}96 \\
96 \\
96\end{array}$ & $\begin{array}{l}0.95 \\
0.95 \\
0.95\end{array}$ & $\begin{array}{l}8 \\
8 \\
8\end{array}$ & $\begin{array}{l}12 \\
12 \\
12\end{array}$ & $\begin{array}{l}- \\
-\end{array}$ & $\begin{array}{l}- \\
-\end{array}$ & $\begin{array}{l}- \\
-\end{array}$ & $\begin{array}{l}27.8 \\
32.7 \\
28.7\end{array}$ & $\begin{array}{l}1.39 \\
1.63 \\
1.43\end{array}$ & $\begin{array}{l}0.05 \\
3.40 \\
0.09\end{array}$ & $\begin{array}{r}\mathbf{0} \\
\mathbf{8} \\
\mathbf{3 0}\end{array}$ & $\begin{array}{l}58 \\
58 \\
58\end{array}$ & $\begin{array}{l}92 / 6 \\
92 / 6 \\
92 / 6\end{array}$ \\
\hline $\begin{array}{l}1.06 \\
1.06 \\
1.06\end{array}$ & $\begin{array}{l}100 \\
100 \\
100\end{array}$ & $\begin{array}{l}50 \\
50 \\
50\end{array}$ & - & $\begin{array}{l}104 \\
104 \\
104\end{array}$ & $\begin{array}{l}107 \\
107 \\
107\end{array}$ & $\begin{array}{l}0.95 \\
0.95 \\
0.95\end{array}$ & $\begin{array}{l}8 \\
8 \\
8\end{array}$ & $\begin{array}{l}12 \\
12 \\
12\end{array}$ & $\begin{array}{l}- \\
-\end{array}$ & $\begin{array}{l}- \\
-\end{array}$ & $\begin{array}{l}- \\
-\end{array}$ & $\begin{array}{l}27.8 \\
35.3 \\
29.9\end{array}$ & $\begin{array}{l}1.39 \\
1.76 \\
1.49\end{array}$ & $\begin{array}{l}0.05 \\
4.10 \\
2.50\end{array}$ & $\begin{array}{r}\mathbf{0} \\
\mathbf{8} \\
\mathbf{3 0}\end{array}$ & $\begin{array}{l}58 \\
58 \\
58\end{array}$ & $\begin{array}{l}92 / 6 \\
92 / 6 \\
92 / 6\end{array}$ \\
\hline $\begin{array}{l}0.27 \\
0.27 \\
0.27\end{array}$ & $\begin{array}{l}- \\
-\end{array}$ & $\begin{array}{l}\mathbf{5 0} \\
\mathbf{5 0} \\
\mathbf{5 0}\end{array}$ & $\begin{array}{l}- \\
-\end{array}$ & $\begin{array}{l}86 \\
86 \\
86\end{array}$ & $\begin{array}{l}71 \\
71 \\
71\end{array}$ & $\begin{array}{l}0.95 \\
0.95 \\
0.95\end{array}$ & $\begin{array}{l}8 \\
8 \\
8\end{array}$ & $\begin{array}{l}12 \\
12 \\
12\end{array}$ & $\begin{array}{l}- \\
-\end{array}$ & $\begin{array}{l}- \\
-\end{array}$ & $\begin{array}{l}- \\
-\end{array}$ & $\begin{array}{l}27.8 \\
35.6 \\
30.0\end{array}$ & $\begin{array}{l}1.39 \\
1.78 \\
1.50\end{array}$ & $\begin{array}{l}0.01 \\
0.07 \\
0.36\end{array}$ & $\begin{array}{r}\mathbf{0} \\
8 \\
\mathbf{3 0}\end{array}$ & $\begin{array}{l}58 \\
58 \\
58\end{array}$ & $\begin{array}{l}92 / 6 \\
92 / 6 \\
92 / 6\end{array}$ \\
\hline $\begin{array}{l}0.37 \\
0.37 \\
0.37\end{array}$ & $\begin{array}{l}6 \\
6 \\
6\end{array}$ & $\begin{array}{l}50 \\
50 \\
50\end{array}$ & $\begin{array}{l}- \\
-\end{array}$ & $\begin{array}{l}36 \\
36 \\
36\end{array}$ & $\begin{array}{l}101 \\
101 \\
101\end{array}$ & $\begin{array}{l}0.95 \\
0.95 \\
0.95\end{array}$ & $\begin{array}{l}8 \\
8 \\
8\end{array}$ & $\begin{array}{l}12 \\
12 \\
12\end{array}$ & $\begin{array}{l}- \\
-\end{array}$ & $\begin{array}{l}- \\
-\end{array}$ & $\begin{array}{l}- \\
-\end{array}$ & $\begin{array}{l}27.8 \\
35.7 \\
30.0\end{array}$ & $\begin{array}{l}1.39 \\
1.78 \\
1.50\end{array}$ & $\begin{array}{l}0.04 \\
0.23 \\
0.12\end{array}$ & $\begin{array}{r}0 \\
8 \\
\mathbf{3 0}\end{array}$ & $\begin{array}{l}58 \\
58 \\
58\end{array}$ & $\begin{array}{l}92 / 6 \\
92 / 6 \\
92 / 6\end{array}$ \\
\hline
\end{tabular}


Table 5. Continued

\begin{tabular}{|c|c|c|c|c|c|c|c|c|c|c|c|c|c|c|c|c|c|}
\hline \multicolumn{4}{|c|}{ Water Chemistry } & \multicolumn{2}{|c|}{ Potentials } & \multirow{2}{*}{$\begin{array}{l}\text { Load } \\
\text { Ratio }\end{array}$} & \multirow[b]{2}{*}{$\begin{array}{r}\text { Freq.. } \\
10^{-2} \mathrm{~Hz}\end{array}$} & \multirow{2}{*}{$\begin{array}{l}\text { Rise } \\
\text { Time. } \\
\text { s }\end{array}$} & \multicolumn{3}{|c|}{ Type 316NG SS } & \multicolumn{4}{|c|}{ Type 304 SS } & \multirow[b]{2}{*}{$\begin{array}{l}\text { Ref. } \\
\text { No. }\end{array}$} & \multirow{2}{*}{$\begin{array}{c}\text { ANL } \\
\text { Report } \\
\text { No. }\end{array}$} \\
\hline $\begin{array}{l}\text { Cond., } \\
\text { is cm-1 }\end{array}$ & $\begin{array}{l}\mathrm{SO}_{4}^{2-} \\
\mathrm{ppb}\end{array}$ & $\begin{array}{l}\mathrm{CrO}_{4}^{2-} \\
\mathrm{ppb}\end{array}$ & $\begin{array}{l}\text { Other. } \\
\text { ppb }\end{array}$ & $\begin{array}{l}304 \text { SS, } \\
\text { mV(SHE) }\end{array}$ & Pt. & & & & $\begin{array}{l}K_{\max }{ }^{d} \cdot \\
M P a \cdot m^{1}\end{array}$ & $\begin{array}{l}\Delta K_{.} e \\
1 / 2\end{array}$ & $\begin{array}{c}\text { Rate. } \\
10^{-10} \mathrm{~m} \cdot \mathrm{s}^{-1}\end{array}$ & $\begin{array}{r}K_{\max }{ }^{d} . \\
\text { MPa. }\end{array}$ & $\begin{array}{l}\Delta K_{0} e \\
\mathrm{~m}^{1 / 2}\end{array}$ & $\begin{array}{c}\text { Rate.s } \\
10^{-10} \mathrm{~m} \cdot \mathrm{s}^{-1}\end{array}$ & $\begin{array}{c}\text { EPR, } \\
\text { C } \mathrm{cm}^{-2}\end{array}$ & & \\
\hline $\begin{array}{l}0.44 \\
0.44 \\
0.44\end{array}$ & $\begin{array}{l}15 \\
15 \\
15\end{array}$ & $\begin{array}{l}50 \\
50 \\
50\end{array}$ & $\begin{array}{l}\bar{z} \\
\overline{-}\end{array}$ & $\begin{array}{l}37 \\
37 \\
37\end{array}$ & $\begin{array}{l}107 \\
107 \\
107\end{array}$ & $\begin{array}{l}0.95 \\
0.95 \\
0.95\end{array}$ & $\begin{array}{l}8 \\
8 \\
8\end{array}$ & $\begin{array}{l}12 \\
12 \\
12\end{array}$ & $\bar{z}$ & $\overline{-}$ & $\begin{array}{l}- \\
\overline{-}\end{array}$ & $\begin{array}{l}27.9 \\
36.2 \\
30.1\end{array}$ & $\begin{array}{l}1.39 \\
1.81 \\
1.50\end{array}$ & $\begin{array}{l}0.06 \\
0.28 \\
0.11\end{array}$ & $\begin{array}{r}0 \\
8 \\
30\end{array}$ & $\begin{array}{l}58 \\
58 \\
58\end{array}$ & $\begin{array}{l}92 / 6 \\
92 / 6 \\
92 / 6\end{array}$ \\
\hline $\begin{array}{l}1.70 \\
1.70 \\
1.70\end{array}$ & $\begin{array}{l}100 \\
100 \\
100\end{array}$ & $\begin{array}{l}200 \\
200 \\
200\end{array}$ & $\begin{array}{l}- \\
-\end{array}$ & $\begin{array}{l}108 \\
108 \\
108\end{array}$ & $\begin{array}{l}142 \\
142 \\
142\end{array}$ & $\begin{array}{l}0.95 \\
0.95 \\
0.95\end{array}$ & $\begin{array}{l}8 \\
8 \\
8\end{array}$ & $\begin{array}{l}12 \\
12 \\
12\end{array}$ & $\begin{array}{l}- \\
-\end{array}$ & $\begin{array}{l}- \\
-\end{array}$ & $\begin{array}{l}- \\
\overline{-}\end{array}$ & $\begin{array}{l}27.9 \\
37.9 \\
30.7\end{array}$ & $\begin{array}{l}1.39 \\
1.89 \\
1.53\end{array}$ & $\begin{array}{l}0.05 \\
3.00 \\
1.70\end{array}$ & $\begin{array}{r}0 \\
8 \\
30\end{array}$ & $\begin{array}{l}58 \\
58 \\
58\end{array}$ & $\begin{array}{l}92 / 6 \\
92 / 6 \\
92 / 6\end{array}$ \\
\hline $\begin{array}{l}1.32 \\
1.32 \\
1.32\end{array}$ & $\begin{array}{l}50 \\
50 \\
50\end{array}$ & $\begin{array}{l}200 \\
200 \\
200\end{array}$ & $\begin{array}{l}- \\
-\end{array}$ & $\begin{array}{l}14 \\
14 \\
14\end{array}$ & $\begin{array}{l}39 \\
39 \\
39\end{array}$ & $\begin{array}{l}0.95 \\
0.95 \\
0.95\end{array}$ & $\begin{array}{l}8 \\
8 \\
8\end{array}$ & $\begin{array}{l}12 \\
12 \\
12\end{array}$ & - & - & $\begin{array}{l}- \\
-\end{array}$ & $\begin{array}{l}27.9 \\
38.2 \\
30.9\end{array}$ & $\begin{array}{l}1.39 \\
1.91 \\
1.54\end{array}$ & $\begin{array}{l}0.05 \\
0.18 \\
0.18\end{array}$ & $\begin{array}{r}0 \\
8 \\
30\end{array}$ & $\begin{array}{l}58 \\
58 \\
58\end{array}$ & $\begin{array}{l}92 / 6 \\
92 / 6 \\
92 / 6\end{array}$ \\
\hline
\end{tabular}

aCompact-tension specimens (1TCT) of Type 316NG SS (Heat No. P91576) and Type 304 SS (Heat No. 30956) with the following heat treatments:

solution anneal at $1050^{\circ} \mathrm{C}$ for $0.5 \mathrm{~h}$ plus $650^{\circ} \mathrm{C}$ for $24 \mathrm{~h}$ for the Type $316 \mathrm{NG}$ SS (EPR $\left.=0 \mathrm{C} \cdot \mathrm{cm}^{-2}\right)$. and solution anneal at $1050^{\circ} \mathrm{C}$ for $0.5 \mathrm{~h}\left(\mathrm{EPR}=0 \mathrm{C} \cdot \mathrm{cm}{ }^{-2}\right.$ ) followed by $700^{\circ} \mathrm{C}$ for $0.25 \mathrm{~h}$ plus $500^{\circ} \mathrm{C}$ for $24 \mathrm{~h}\left(\mathrm{ElPR}=2 \mathrm{C} \cdot \mathrm{cm}^{-2}\right) .700^{\circ} \mathrm{C}$ for $0.67 \mathrm{~h}\left(\mathrm{EPR}=8 \mathrm{C} \cdot \mathrm{cm}^{-2}\right) .700^{\circ} \mathrm{C}$ for $12 \mathrm{~h}\left(\mathrm{EPR}=20 \mathrm{C} \cdot \mathrm{cm}^{-2}\right)$. or $700^{\circ} \mathrm{C}$ for $24 \mathrm{~h}$ $\left(E P R=30 \mathrm{C} \cdot \mathrm{cm}^{-2}\right)$ for the Type 304 SS.

bEffluent DO concentration was 200-300 ppb; feedwater oxygen concentration was approximately a factor of 2 higher to compensate for oxygen depletion by corrosion of the autoclave systems.

CPositive sawtooth waveform was used.

dStress intensity. $K_{\max }$. values at the end of $a=1000-\mathrm{h}$ time period of steady-state crack growth.

$e_{\Delta K}=K_{\max }(1-R)$, where the load ratio $R=K_{\min } / K_{\max }$.

fracking was transgranular, i.c., TGSCC.

BCracking was intergranular, i.c., IGSCC.

h Compact-tension specimens (0.7TCT) of Type 316NG SS (I lcat No. 12440104) with the following heat treatment: solution anncal at $1050^{\circ} \mathrm{C}$ for $0.5 \mathrm{~h}$ plus $650^{\circ} \mathrm{C}$ for $24 \mathrm{~h}\left(\mathrm{EPR}=0 \mathrm{C} \cdot \mathrm{cm}^{-2}\right)$.

isine wavelorm was used. 
Table 6. Summary of crack growth results for Type 347 SS specimens ${ }^{a}$ in axygenated water at $289^{\circ} \mathrm{C}$, in which load ratiob and stress intensity were varled

\begin{tabular}{|c|c|c|c|c|c|c|c|c|c|c|c|c|}
\hline \multicolumn{3}{|c|}{ Water Chemistry } & \multicolumn{2}{|c|}{ Potentials } & \multirow{2}{*}{$\begin{array}{l}\text { load } \\
\text { Ratio }\end{array}$} & \multirow[b]{2}{*}{$\begin{array}{l}\text { Preq.. } \\
10^{-2} \mathrm{~Hz}\end{array}$} & \multirow{2}{*}{$\begin{array}{l}\text { Rise } \\
\text { Time. } \\
\text { s }\end{array}$} & \multicolumn{3}{|c|}{ CaR Data } & \multirow[b]{2}{*}{$\begin{array}{l}\text { Ref. } \\
\text { No. }\end{array}$} & \multirow{2}{*}{$\begin{array}{c}\text { ANL } \\
\text { Report } \\
\text { No. }\end{array}$} \\
\hline $\begin{array}{l}\text { Cond.. } \\
\mu S \cdot \mathrm{cm}^{-1}\end{array}$ & $\begin{array}{c}\mathrm{O}_{2} \\
\mathrm{ppb}\end{array}$ & $\begin{array}{l}\mathrm{so}_{4}^{2-} \\
\mathrm{ppb}\end{array}$ & $\begin{array}{r}304 \text { SS. } \\
\text { mV(s }\end{array}$ & Pt, & & & & $\begin{array}{l}\mathrm{K}_{\mathrm{max}} \mathrm{c} \cdot \\
\mathrm{MPa} \cdot \mathrm{n}\end{array}$ & $\Delta K_{i}^{d}$ & $\begin{array}{l}\text { Rate, } \\
10 \cdot 10 \mathrm{~m} \cdot \mathrm{g}^{-1}\end{array}$ & & \\
\hline \multicolumn{13}{|c|}{ slow-Cooled Specimen } \\
\hline $\begin{array}{l}0.92 \\
0.92 \\
0.95 \\
0.96 \\
0.92 \\
0.89\end{array}$ & $\begin{array}{l}200 \\
200 \\
200 \\
200 \\
200 \\
200\end{array}$ & $\begin{array}{l}100 \\
100 \\
100 \\
100 \\
100 \\
100\end{array}$ & $\begin{array}{l}147 \\
121 \\
174 \\
185 \\
176 \\
138\end{array}$ & $\begin{array}{l}200 \\
159 \\
223 \\
211 \\
210 \\
174\end{array}$ & $\begin{array}{l}0.90 \\
0.90 \\
0.95 \\
0.95 \\
0.95 \\
1.00\end{array}$ & $\begin{array}{l}8 \\
8 \\
8 \\
8 \\
8 \\
0\end{array}$ & $\begin{array}{c}12 \\
12 \\
12 \\
12 \\
12 \\
\infty\end{array}$ & $\begin{array}{l}19.9 \\
22.3 \\
22.4 \\
27.6 \\
30.7 \\
31.1\end{array}$ & $\begin{array}{l}1.99 \\
2.23 \\
1.12 \\
1.38 \\
1.53 \\
0\end{array}$ & $\begin{array}{l}0.85 \\
3.40 \\
0.09 \\
1.70 \\
3.00 \\
0.89\end{array}$ & $\begin{array}{l}62 \\
62 \\
62 \\
62 \\
62 \\
62\end{array}$ & $\begin{array}{l}90 / 48 \\
90 / 48 \\
90 / 48 \\
90 / 48 \\
90 / 48 \\
90 / 48\end{array}$ \\
\hline \multicolumn{13}{|c|}{ Waler-Quenched Specinen } \\
\hline $\begin{array}{l}0.93 \\
0.92 \\
0.92 \\
0.95 \\
0.96 \\
0.92\end{array}$ & $\begin{array}{l}200 \\
200 \\
200 \\
200 \\
200 \\
200\end{array}$ & $\begin{array}{l}100 \\
100 \\
100 \\
100 \\
100 \\
100\end{array}$ & $\begin{array}{l}150 \\
147 \\
121 \\
174 \\
185 \\
176\end{array}$ & $\begin{array}{l}208 \\
200 \\
159 \\
223 \\
211 \\
210\end{array}$ & $\begin{array}{l}0.50 \\
0.90 \\
0.90 \\
0.95 \\
0.95 \\
0.95\end{array}$ & $\begin{array}{l}8 \\
8 \\
8 \\
8 \\
8 \\
8\end{array}$ & $\begin{array}{l}12 \\
12 \\
12 \\
12 \\
12 \\
12\end{array}$ & $\begin{array}{l}19.8 \\
19.8 \\
22.0 \\
22.1 \\
27.0 \\
29.8\end{array}$ & $\begin{array}{l}9.90 \\
1.98 \\
2.20 \\
1.11 \\
1.35 \\
1.49\end{array}$ & $\begin{array}{c}30.0 \\
0.09 \\
1.30 \\
0.08 \\
0.60 \\
0.60\end{array}$ & $\begin{array}{l}62 \\
62 \\
62 \\
62 \\
62 \\
62\end{array}$ & $\begin{array}{l}90 / 48 \\
90 / 48 \\
90 / 48 \\
90 / 48 \\
90 / 48 \\
90 / 48\end{array}$ \\
\hline 0.89 & 200 & 100 & 138 & 174 & 1.00 & 0 & $\infty$ & 30.0 & 0 & 0.61 & 62 & $90 / 48$ \\
\hline
\end{tabular}

acompact-tension specimens (19Cl) were labricated from Ileat No. 46113.

bpositive sawtooth waveform was used.

CStress intensity, $K_{\max }$ value at the end of each time pertod.

$d_{\Delta K}=K_{\max }(1-R)$, where the load ratio $R=K_{1 \text { inin }} / K_{m \text { max }}$.

Table 7. Summary of crack growth results for cast SS specimens $a$ in water at $289^{\circ} \mathrm{C}$, in which load ratio ${ }^{b}$ and stress intensity were varied 7

\begin{tabular}{|c|c|c|c|c|c|c|c|c|c|c|c|}
\hline \multicolumn{3}{|c|}{ Water Cheinistry } & \multicolumn{2}{|c|}{ Potentials } & \multirow{2}{*}{$\begin{array}{l}\text { load } \\
\text { Ratios }\end{array}$} & \multirow[b]{2}{*}{$\begin{array}{l}\text { Freq.. } \\
10^{-2} \mid 1 \%\end{array}$} & \multirow{2}{*}{$\begin{array}{c}\text { Rise } \\
\text { Tine. } \\
\text { s }\end{array}$} & \multicolumn{3}{|c|}{ CGR Data } & \multirow{2}{*}{$\begin{array}{c}\text { ANL } \\
\text { Report } \\
\text { No. }\end{array}$} \\
\hline $\begin{array}{l}\text { Cond. } \\
\mu \text { s.cm-1 }\end{array}$ & $\begin{array}{c}\mathrm{O}_{2} . \\
\text { ppin }\end{array}$ & $\begin{array}{l}\mathrm{SO}_{4}^{2} \\
\mathrm{ppb}\end{array}$ & $\begin{array}{r}304 \mathrm{~s} \\
\mathrm{mV}\end{array}$ & PIE) & & & & $\begin{array}{r}K_{\text {max }} \\
M P_{i}\end{array}$ & $\ln ^{\Delta / 2}$ & $\begin{array}{c}\text { Rate, } \\
10^{-10} \mathrm{~m} \cdot \mathrm{s}^{-1}\end{array}$ & \\
\hline \multicolumn{12}{|c|}{ As-recetved CF-3M Spxecincon (Ileat No. P5524) Containing 5.0\% Ferrite } \\
\hline 0.90 & 0.2 & 100 & 136 & 120 & 0.95 & 7.7 & 12 & 22.6 & 1.13 & 1.90 & $90 / 4$ \\
\hline 0.90 & 0.2 & 100 & 106 & (36 & $0.25^{c}$ & 10.0 & 5 & 20.7 & 15.50 & 35.0 & $90 / 4$ \\
\hline 0.90 & 0.2 & 100 & 81 & 41 & 0.95 & 7.7 & 12 & 24.0 & 1.20 & 2.50 & $90 / 4$ \\
\hline 1.10 & 0.2 & 100 & 134 & 95 & 1.00 & 0 & $\infty$ & 24.1 & 0 & 0.32 & $90 / 4$ \\
\hline \multicolumn{12}{|c|}{ As-recelved Cl-3 Specimen (1lcat No. P2) Contatning $15.6 \%$ Ferrite } \\
\hline 0.93 & 0.2 & 100 & 150 & 208 & $0.50^{\circ}$ & 7.7 & 12 & 19.6 & 9.80 & 27.0 & $90 / 48$ \\
\hline 0.92 & 0.2 & 100 & 147 & 200 & 0.90 & 7.7 & 12 & 19.7 & 1.97 & 0.09 & $90 / 48$ \\
\hline 0.92 & 0.2 & 100 & 121 & 159 & 0.90 & 7.7 & 12 & 21.8 & 2.18 & 0.40 & $90 / 48$ \\
\hline 0.92 & 0.2 & 100 & 176 & 210 & 0.95 & 7.7 & 12 & 29.2 & 1.46 & 0.11 & $90 / 48$ \\
\hline \multicolumn{12}{|c|}{ As-received CF-8M Spxecimen (Ileat No. } \\
\hline 0.13 & 6.2 & 0 & 258 & 332 & 0.95 & 7.7 & 12 & 25.3 & 1.26 & $<0.02$ & $93 / 2$ \\
\hline 0.16 & 5.3 & 0 & 240 & 317 & 0.95 & 7.7 & 12 & 34.7 & 1.73 & 1.6 & $93 / 2$ \\
\hline 0.14 & 7.0 & 0 & 242 & 296 & 0.95 & 7.7 & 12 & 35.2 & 1.76 & 1.8 & $93 / 2$ \\
\hline 0.13 & $<0.002$ & 0 & -560 & -580 & 0.95 & 7.7 & 12 & 35.2 & 1.76 & $<0.02$ & $93 / 2$ \\
\hline 0.18 & 0.3 & 0 & 198 & 243 & 0.95 & 7.7 & 12 & 36.4 & 1.82 & 1.4 & $93 / 2$ \\
\hline 0.11 & 1.1 & 0 & 216 & 260 & 0.95 & 7.7 & 12 & 36.8 & 1.84 & 1.5 & $93 / 2$ \\
\hline 0.11 & 0.9 & 0 & 206 & 257 & 1.0 & 0 & $\infty$ & 36.8 & 0 & $<0.02$ & $93 / 2$ \\
\hline 0.10 & 7.2 & 0 & 247 & 301 & 0.95 & 7.7 & 12 & 45.6 & 2.28 & 2.5 & $93 / 2$ \\
\hline
\end{tabular}


Table 7. Continued

\begin{tabular}{|c|c|c|c|c|c|c|c|c|c|c|c|}
\hline \multicolumn{3}{|c|}{ Water Chemistry } & \multicolumn{2}{|c|}{ Potentials } & \multirow{2}{*}{$\begin{array}{l}\text { Leind } \\
\text { Ratio }\end{array}$} & \multirow[b]{2}{*}{$\begin{array}{l}\text { Fruq.. } \\
1021 \mathrm{k} .\end{array}$} & \multirow{2}{*}{$\begin{array}{l}\text { Rise } \\
\text { Time. } \\
\text { s }\end{array}$} & \multicolumn{3}{|c|}{ Cor Data } & \multirow{2}{*}{$\begin{array}{c}\text { ANL } \\
\text { Peport } \\
\text { No. }\end{array}$} \\
\hline $\begin{array}{l}\text { Cond. } \\
\mu \mathrm{s} \cdot \mathrm{cm}^{-1}\end{array}$ & $\begin{array}{ll}\mathrm{O}_{2}, & \mathrm{~s} \\
\mathrm{ppm} & \mathrm{s}\end{array}$ & $\begin{array}{l}\text { soon. } \\
\text { ppb }\end{array}$ & $\begin{array}{l}304 \mathrm{ss} \\
\mathrm{mV} / \mathrm{s}\end{array}$ & $\begin{array}{l}\text { PA. } \\
\text { [IE) }\end{array}$ & & & & $\begin{array}{r}K_{\text {max }}{ }^{c} \\
M P_{a}\end{array}$ & $\begin{array}{l}\Delta K, d \\
n 1 / 2\end{array}$ & $\begin{array}{c}\text { Rate, } \\
10 \cdot 10 \mathrm{~m} \cdot \mathrm{s}^{-1}\end{array}$ & \\
\hline \multicolumn{12}{|c|}{ Agedl CF-8M specimen (lileat No. 75) Containing 27.8\% Ferrite } \\
\hline 0.13 & 6.2 & 0 & 258 & 332 & 0.95 & 7.7 & 12 & 24.7 & 1.23 & 4.4 & $93 / 2$ \\
\hline 0.16 & 5.3 & 0 & 240 & 317 & 0.95 & 7.7 & 12 & 37.2 & 1.86 & 8.1 & $93 / 2$ \\
\hline 0.14 & $7 n$ & 0 & 242 & 296 & 0.95 & 7.7 & 12 & 46.0 & 2.30 & 18.6 & $93 / 2$ \\
\hline 0.13 & $<0.0020$ & & -560 & -580 & 0.95 & 7.7 & 12 & 46.0 & 2.30 & $<0.02$ & $93 / 2$ \\
\hline 0.18 & 0.3 & 0 & 198 & 243 & 0.95 & 7.7 & 12 & 47.5 & 2.38 & 1.1 & $93 / 2$ \\
\hline 0.11 & 1.1 & 0 & 216 & 260 & 0.95 & 7.7 & 12 & 53.6 & 2.68 & 7.3 & $93 / 2$ \\
\hline 0.11 & 0.9 & 0 & 206 & 257 & 1.0 & 0 & $\infty$ & 61.4 & 0 & 20.8 & $93 / 2$ \\
\hline
\end{tabular}

\begin{tabular}{|c|c|c|c|c|c|c|c|c|c|c|c|}
\hline & & & & Ageds CF-HM & Spetc: & $n$ llk & No. 75) & Containtn & $27.8 \%$ & Ferrite & \\
\hline 0.07 & 4.4 & 0 & 256 & 280 & 0.80 & 7.7 & 12 & 31.8 & 6.36 & 58.0 & $93 / 27$ \\
\hline 0.08 & 4.3 & 0 & 237 & 243 & 0.90 & 7.7 & 12 & 33.1 & 3.31 & 16.2 & $93 / 27$ \\
\hline 0.07 & 4.2 & 0 & 247 & 301 & 0.70 & 7.7 & 12 & 34.9 & 10.47 & 122.0 & $93 / 27$ \\
\hline 0.07 & 4.5 & 0 & 252 & 3.37 & 0.30 & 7.7 & 12 & 38.2 & 26.74 & 349.0 & $93 / 27$ \\
\hline 0.11 & 0.43 & 0 & 205 & 218 & 0.90 & 7.7 & 12 & 40.6 & 4.06 & 15.9 & $93 / 27$ \\
\hline 0.07 & 0.60 & 0 & 214 & 223 & 0.70 & 7.7 & 12 & 43.8 & 13.14 & 110.0 & $93 / 27$ \\
\hline 0.11 & 0.50 & 0 & 205 & 211 & 0.30 & 7.7 & 12 & 52.5 & 36.75 & 666.0 & $3 / 27$ \\
\hline
\end{tabular}

\begin{tabular}{|c|c|c|c|c|c|c|c|c|c|c|c|}
\hline \multirow[b]{2}{*}{0.07} & \multirow[b]{2}{*}{4.4} & \multicolumn{10}{|c|}{ As-recelved (F-8 Spxcimen (I leat No. 68) Contalning 27.8\% Ferrite } \\
\hline & & 0 & 256 & 280 & 0.80 & 7.7 & 12 & 30.2 & 6.04 & 16.9 & $93 / 27$ \\
\hline 0.08 & 4.3 & 0 & 237 & 24.3 & 0.90 & 7.7 & 12 & 30.5 & 3.05 & 3.9 & 93/27 \\
\hline 0.07 & 4.2 & 0 & 247 & 301 & 0.70 & 7.7 & 12 & 30.9 & 9.27 & 39.2 & $93 / 27$ \\
\hline 0.07 & 4.5 & 0 & 252 & 337 & 0.30 & 7.7 & 12 & 32.0 & 22.40 & 170.0 & $93 / 27$ \\
\hline 0.11 & 0.43 & 0 & 205 & 218 & 0.90 & 7.7 & 12 & 32.4 & 3.24 & 2.9 & $93 / 27$ \\
\hline 0.07 & 0.60 & 0 & 214 & 223 & 0.70 & 7.7 & 12 & 33.1 & 9.93 & 32.8 & $93 / 27$ \\
\hline 0.11 & 0.50 & 0 & 205 & 211 & 0.30 & 7.7 & 12 & 34.4 & 24.08 & 157.0 & $93 / 27$ \\
\hline
\end{tabular}

\begin{tabular}{|c|c|c|c|c|c|c|c|c|c|c|c|}
\hline \multirow[b]{2}{*}{0.13} & \multicolumn{11}{|c|}{ Agedf CF-8 Spo:imen (I leat No. (68) Containing 27.8\% Ferrite } \\
\hline & 6.2 & 0 & 258 & 3332 & 0.95 & 7.7 & 12 & 26.0 & 1.30 & $<0.02$ & $93 / 2$ \\
\hline 0.16 & 5.3 & 0 & 240 & 317 & 0.95 & 7.7 & 12 & 35.0 & 1.75 & 0.42 & $93 / 2$ \\
\hline 0.14 & 7.0 & 0 & 242 & 290 & 0.95 & 7.7 & 12 & 36.9 & 1.85 & 4.6 & $93 / 2$ \\
\hline 0.13 & $<0.0020$ & & $-5(30$ & -580 & 0.95 & 7.7 & 12 & 36.9 & 1.85 & $<0.02$ & $93 / 2$ \\
\hline 0.18 & 0.3 & 0 & 198 & 243 & 0.95 & 7.7 & 12 & 37.4 & 1.87 & 0.93 & $93 / 2$ \\
\hline 0.11 & 1.1 & 0 & 216 & $2(30)$ & 0.95 & 7.7 & 12 & 37.8 & 1.89 & 1.4 & $93 / 2$ \\
\hline 0.11 & 0.9 & 0 & 206 & 257 & 1.0 & 0 & $\infty$ & 37.8 & 0 & $<0.02$ & $93 / 2$ \\
\hline 0.10 & 7.2 & 0 & 247 & 301 & 0.95 & 7.7 & 12 & 52.5 & 2.62 & 11.3 & $93 / 2$ \\
\hline
\end{tabular}

\begin{tabular}{|c|c|c|c|c|c|c|c|c|c|c|c|}
\hline \multirow[b]{2}{*}{0.07} & \multicolumn{11}{|c|}{ Akede (F-8 Sperinicu lllent No. (is) Contatning 27.8\% Ferrte } \\
\hline & 4.4 & 0 & 256 & 280 & 0.80 & 7.7 & 12 & 30.9 & 6.18 & 31.9 & $93 / 27$ \\
\hline 0.08 & 4.3 & 0 & $2: 37$ & 243 & 0.90 & 7.7 & 12 & 31.3 & 3.13 & 5.4 & $93 / 27$ \\
\hline 0.07 & 4.2 & 0 & 247 & 301 & 0.70 & 7.7 & 12 & 32.1 & 9.63 & 53.7 & $93 / 27$ \\
\hline 0.07 & 4.5 & o) & 252 & 337 & 0.30 & 7.7 & 12 & 33.0 & 23.10 & 157.0 & $93 / 27$ \\
\hline 0.11 & 0.43 & 0 & 205 & 218 & 0.90 & 7.7 & 12 & 33.6 & 3.36 & 4.2 & $93 / 27$ \\
\hline 0.07 & 0.60 & 0 & 214 & 223 & 0.70 & 7.7 & 12 & 34.4 & 10.32 & 32.4 & $93 / 27$ \\
\hline 0.11 & 0.50 & () & 205 & 211 & 0.350 & 7.7 & 12 & 35.8 & 25.06 & 191.0 & $93 / 27$ \\
\hline
\end{tabular}

aCompact-tension specimens werc fabricated from Ileat No. F5524 (0.7TCT) and (1TCT) from Heat Nos. P2. 68, and 75.

bPositive sawtooth waveform was used.

CStress intensity, $K_{t n a x}$, valuc at the end of each time period.

$d_{\Delta K}=K_{\text {max }}(1-R)$, where the load ratio $R=K_{m i n} / K_{m a x}$.

esine waveform was used.

fspecimens aged for $10,000 \mathrm{~h}$ at $400^{\circ} \mathrm{C}$.

Especimens aged fo: $30,000 \mathrm{~h}$ all $350^{\circ} \mathrm{C}$. 


\section{Appendlx B: Effects of Water Chemistry on CGRs of Austenitic Stainless Steols}

The correlations in Eqs. 1-6 are primarily based on DO concentration in water although the CGR data in the Figs. 1-7 were obtained in both HP water $\left(<0.2 \mu S \cdot \mathrm{cm}^{-1}\right)$ and in water containing $\leq 100 \mathrm{ppb}$ of various ionic impurities ( $\left.\leq 1.0 \mu \mathrm{S} \cdot \mathrm{cm}^{-1}\right)$. It is well known that ionic impurities $\left(\mathrm{SO}_{4}^{2-}, \mathrm{Cl}^{-}\right.$. etc.) at low concentrations increase susceptibility of these steels to SCC (decrease the threshold stress intensity for cracking) and also increase the CGRs through a power-law dependence on concentration (e.g.. $\left[\mathrm{SO}_{4}^{2-}\right]^{2}$, where experimental and predicted values of $\mathrm{z}$ are $=0.5$ and 1.8, respectively). 24 To mitigate SCC of sensitized Type 304 SS in BWR recirculation system piping, plant operators have made significant improvements in water quality over the past several years. Because the ingress of tonic impurities into the coolant system from leaks in condenser tubes and from ton-exchange resins (including resin fines) in the reactor water cleanup system (RWCS) is maintained at very low levels, soluble corrosion products from system materials are the major species present in BWR water. Examples include $\mathrm{Cu}^{+}$and $\mathrm{Cu}^{2+}$ in plants with copper alloy condenser tubes and/or feedwater heaters and $\mathrm{HCrO}_{4}^{-}$from corrosion of Types $304 \mathrm{SS}$ feedwater heaters. Type 430 SS molsture separator vanes, and Type 410 SS high-pressure turbine rotor blades. The austenitic SS piping, weld cladding on the interior of the reactor vessel. and internal components fabricated from this material are relatively minor sources of $\mathrm{HCrO}_{4}^{-}$. Because only a small fraction of the recirculation water in BWRs passes through the RWCS, the concentration of corrosion-product lons in the reactor water can be considerably greater than in the leedwater (e.g., $\approx 25-35 \mathrm{ppb}$ versus $<1 \mathrm{ppb} \mathrm{HCrO}_{4}^{-}$, respectively). Consequently, in plants that operate with optimum water chemistry, $\mathrm{HCrO}_{4}^{-}$and the counterbalancing hydrontum cation $\left(\mathrm{H}_{3} \mathrm{O}^{+}\right)$are major contributors to water conductivity. with minor contributions from $\mathrm{SO}_{4}^{2-}$ and other anions.

\section{Role of Chromate and Sulfate in SCC}

We conducted fracture-mechanics CGR experiments to determine the effect of $\mathrm{HCrO}_{4}^{-}$ additions to simulated BWR water (wilhout and with low levels of $\mathrm{SO}_{4}^{2-}$ ) on SCC of Type 304 SS.26 Specimens were heat treated to produce a solution-annealed condition and low, moderate, and high levels of sensitization corresponding to electrochemical potentiokinetic reactivation (EPR) values of $0,2,8$, and $30 \mathrm{C} \cdot \mathrm{cm}^{-2}$, respectively.

The solution-annealed specimen (EPR $=0 \mathrm{C} \cdot \mathrm{cm}^{-2}$ ) exhibited very low CGRs $\left(<5 \times 10^{-12} \mathrm{~m} \cdot \mathrm{s}^{-1}\right)$ under all water chemistry conditions. In the case of the specimens with moderate and high degrees of sensitization (EPR $=8$ and $30 \mathrm{C} \cdot \mathrm{cm}^{-2}$ ), the specimen with the lower EPR value exhibiled the highest CGRs under each test condition. This level of sensitization produced maximum SCC susceptibllity in SSRT tests at $289^{\circ} \mathrm{C}$ in oxygenated water without and with $100 \mathrm{ppb} \mathrm{SO} \mathrm{SO}^{2-} .26$ The results for the sensitized specimens can be summarized. In oxygenated water, $50-200 \mathrm{ppb} \mathrm{HCrO}_{4}^{-}$has a beneficial effect, as indicated by the low CGRs $\left(<7 \times 10^{-11} \mathrm{~m} \cdot \mathrm{s}^{-1}\right)$. In water containing $50 \mathrm{ppb} \mathrm{HCrO}_{4}^{-}$, low levels of $\mathrm{SO}_{4}^{2-}$

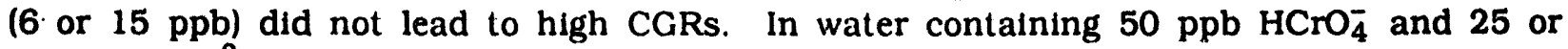
$100 \mathrm{ppb} \mathrm{SO}{ }_{4}^{2-}$. CGRs of the moderately sensilized specimen (EPR $=8 \mathrm{C} \cdot \mathrm{cm}^{-2}$ ) increased significantly (to $>3 \times 10^{-10} \mathrm{~m} \cdot \mathrm{s}^{-1}$ ), whereas the heavily sensitized specimen exhibited this

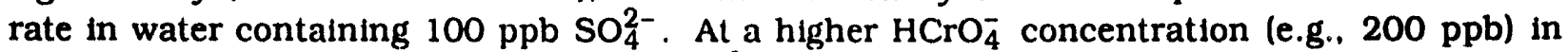
oxygenated water containing $100 \mathrm{ppb} \mathrm{SO}{ }_{4}^{2-}$, the CGRs of the sensitized specimens were high $\left(>1 \times 10^{-10} \mathrm{~m} \cdot \mathrm{s}^{-1}\right)$. However, after decreasing the $\mathrm{SO}_{4}^{2-}$ concentration to $50 \mathrm{ppb}$, the CGRs of the sensitired specimens decreased $10<2 \times 10^{-11} \mathrm{~m} \cdot \mathrm{s}^{-1}$. 
Although SSRT tests indicated that chromate at concentrations $>100 \mathrm{ppb}$ in axygenated water contributes to intergranular (IG) SCC in a manner similar to that of other oxyanions, 24.26 the present CGR results suggest that this species has a mitigating effect on IGSCC at lower concentrations, provided that the ratlo of chromate concentration to sulfate concentration is $\geq 3$. Figure 9 shows the CGRs of the moderately sensitized specimen $\left(E P R=8 \mathrm{C} \cdot \mathrm{cm}^{-2}\right.$ ) as a function of the ratio of the concentrations of chromate and sulfate in the feedwater. For the purpose of obtaining the ratios in Fig. 9, when these specles were not added to the feedwater, their concentrations were assumed to be $\approx 1 \mathrm{ppb}$. The limited data suggest that the CGRs are in the low regime if the $\left(\mathrm{CrO}_{4}^{2-}\right) /\left(\mathrm{SO}_{4}^{2-}\right)$ ratio in the feedwater is $\geq 3$. Chromate concentrations in feedwater and effluent water were determined by colorimetric analyses $\left(\mathrm{Cr}^{+6}\right)$ on grab samples. The measured feedwater concentrations were in excellent agreement with the amounts added to the water; however, the effluent values were lower by a factor of $>5$ because of the low flow velocity and reaction with the large surface area of SS in the autoclave system at $289^{\circ} \mathrm{C}$. The feedwater and effluent sulfate concentrations were virtually the same in all experiments.

To further explore the dependence of CGR on impurity concentration. ANL CGR data for sensitized Type 304 SS were separated into three conductivity regimes, 1.e., 0.88-1.1. $0.28-0.54$, and $<0.2 \mu \mathrm{S} \cdot \mathrm{cm}^{-1}$, and the rates were compared with values obtained from the ASME Section XI correlation lor crack growth in air under the particular loading conditions. The data obtained at load ratios $\geq 0.95$ and $\leq 0.92$ are shown in Figs. 10 and 11 , respectively. The results at the three conductivity levels in water containing $\approx 200 \mathrm{ppb}$ DO fall within a wide scatter band and it is difficult to deduce a clear influence of water purity on the CGRs. Figure 12 shows CGR results for Type 304 SS with different levels of sensitization at an $R$ value of 0.95 in water containing $\approx 200 \mathrm{ppb} \mathrm{DO}$ and different chromate and sulfate concentrations in which the $\left(\mathrm{CrO}_{4}^{2-}\right) /\left(\mathrm{SO}_{4}^{2-}\right)$ ratio was $>3$. The conductivity of the water in these tests was $0.2-1.3 \mu \mathrm{S} \cdot \mathrm{cm}^{-1}$, i.e., the same range as in the data in Figs. 10 and 11 . In contrast to the latter results, the CGRs in Fig. 12 lie near the ASME air line and are an order of magnitude lower than the corrosion-fatigue curve for high-temperature water obtained from Eqs. 1 and 2.

Because chromate concentrations in BWR recirculation water are $\approx 25-35 \mathrm{ppb}$, these results suggest that sulfate concentration would have to be maintained at $<10 \mathrm{ppb}$ to mitigate IGSCC. Typically, values of $\approx 5 \mathrm{ppb}$ can be achieved. Consequently, these water chemistry conditions would place sensitized Type 304 SS in the low-CGR regime. Because laboratory corrosion-fatigue lesting facilities typically employ once-through water flow systems, chromate levels due to corrosion of the autoclave and piping are not high enough to reflect the fotentially beneficial effecl observed when chromate is added to feedwater containing low-sulfate concentrations. Consequently, most CGR data obtained in HP oxygenated water will lie above the air line, as shown in Figs. 10 and 11 . Hence, the laboratory test results, although conservative, may not be representative of CGRs in recirculation piping exposed to a BWR water chemistry with low sulfate levels $(<10 \mathrm{ppb})$ where the chromate level is $\approx 25-35 \mathrm{ppb}$. Water chemistry data that includes both the chromate and sulfate concentration is avallable for a number of BWRs. An assessment of appropriate CGRs for plants that have a $\left(\mathrm{CrO}_{4}^{2-}\right) /\left(\mathrm{SO}_{4}^{2-}\right)$ ratio $>3$ can be made on a case-bycase basis. A factor of 10-20 in the expected CGRs for a shallow crack in the HAZ of a weld could provide significant relief in inspection intervals and influence piping repair and replacement decisions. Also, efforts to remove chromate from recirculation loop water to 

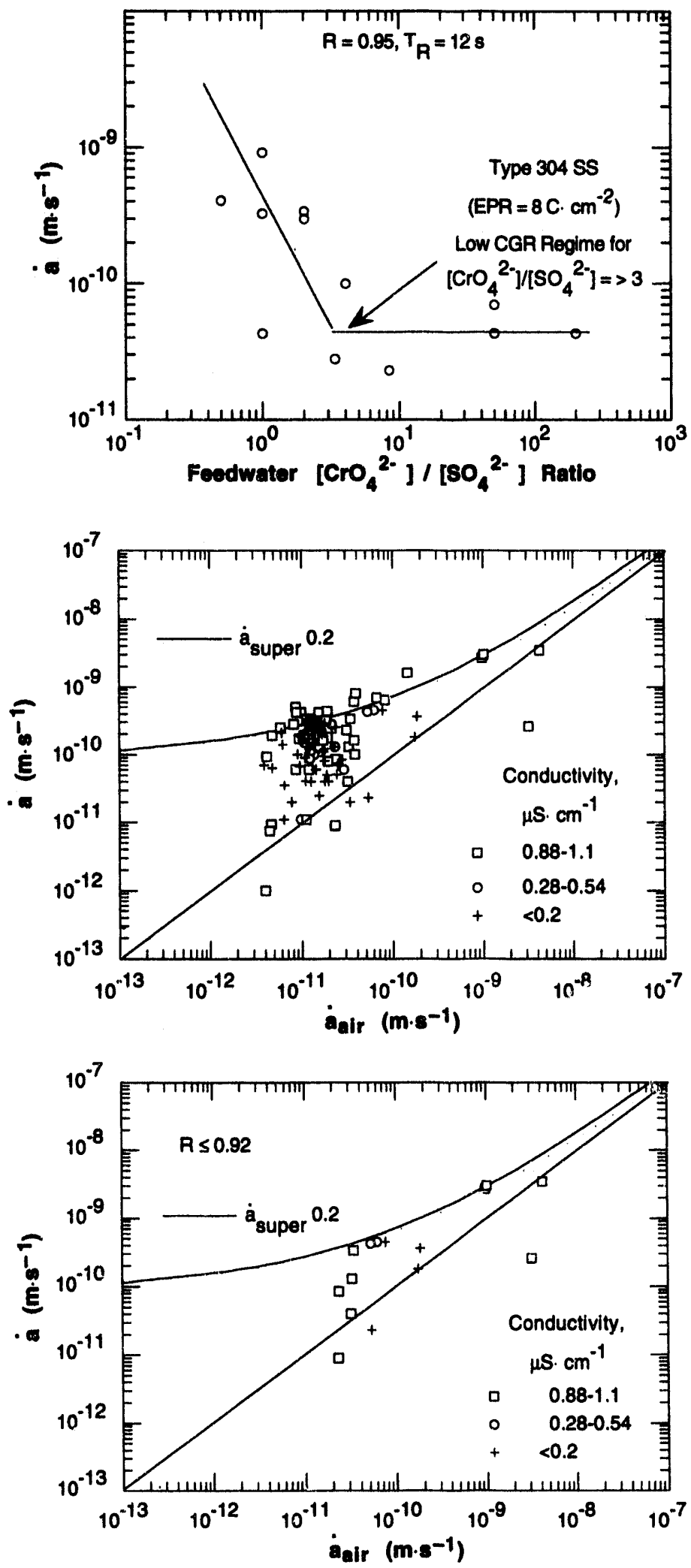

Fig. 9.

Regime of high and low CGRs for moderately sensitized (EPR $=8$ C. $\mathrm{cm}^{-2}$ ) Type 304 SS specimen at $289^{\circ} \mathrm{C}$ as a function of ratio of chromate and sulfate concentrations in feedwater containing $\approx 200$ ppb DO

Fig. 10.

ANL corrosion fatigue data at $R \geq 0.95$ for sensitized Type 304 SS in $289^{\circ} \mathrm{C}$ water containing 200 ppb DO at several conductivity levels. Diagonal line corresponds to crack growth in air.
Fig. 11.

ANL corrosion fatigue data at $R \leq 0.92$ for sensitized Type 304 SS in $289^{\circ} \mathrm{C}$ water containing 200 ppb DO at several conductivity levels. Diagonal line corresponds to crack growth in air. 


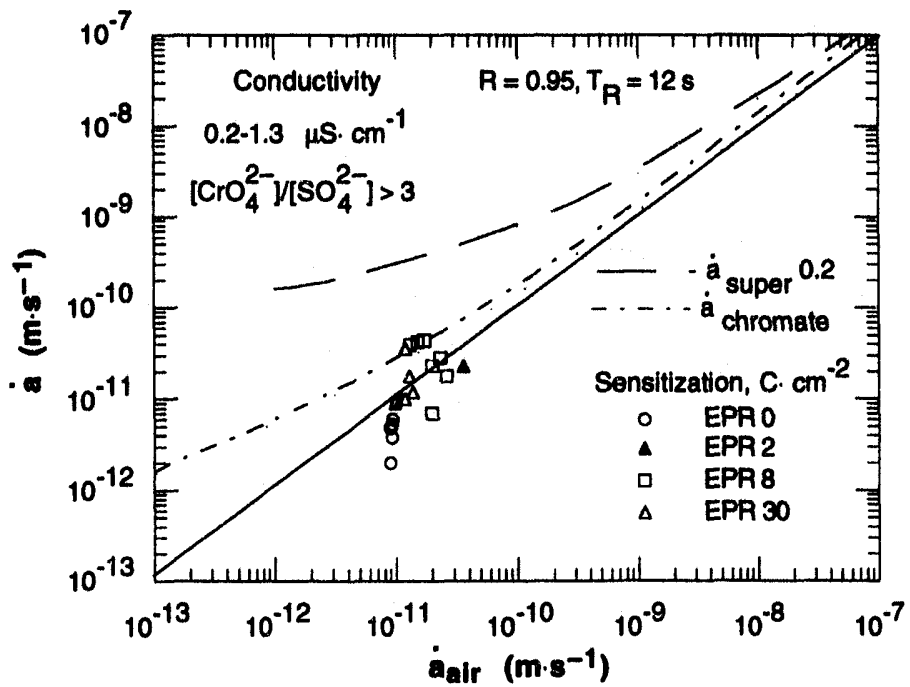

Fig. 12.

Corrosion fatigue data in water containing 200 ppb DO and chromate and sulfate at low concentrations where the $\left.\left[\mathrm{CrO}_{4}^{2-}\right] / \mathrm{SO}_{4}^{2-}\right]=>3$. Diagonal line corresponds to crack growth in air.

decrease the water conductivity and mitigate SCC (without decreasing the critical sulfate level) would be counterproductive.

\section{Effect of Organic Substances in Oxygenated Water on SCC}

Typical chemicals at power plants include paint products, glycol, hydraulic fluids, lubricants, detergents, chemical cleaners, laundry chemicals, freons, diesel fuel, and tonexchange resin regeneration chemicals. Potential chemical contaminants 27 and possible pathways $^{28}$ for entry of various substances into BWR coolant systems have been evaluated. Some of the long-lived products that may exist in BWR water due to organic intrusions are carboxylic acids, alcohols, phenolics, aromatic hydrocarbons, hydrogen halides, sulfuric and sulfonic acids, amines, and other substances. 27 These organic impurities and their decomposition products are a potential concern in BWR water and PWR secondary-system water in terms of increased susceptibility to localized corrosion and SCC of piping and heatexchanger tube materials.

Organic impurities are also a concern in PWR secondary-coolant water systems because organic acids increase cation conductivity, which complicates secondary water monitoring and control. A survey of organic acids, total organic carbon, and inorganic anions in the secondary water cycles of 13 PWRs indicated that organic acids were responsible for a major fraction of the cation conductivity in many of the plants. ${ }^{29}$ Acetic and formic were the most common acids; however, lactic, propionic, and butyric acids were also present in some of the systems. Make-up water was the major source of the organic impurities, some of which were in colloidal, nonionic form. ${ }^{29}$

The role of organic oxygen scavengers and other organic species in SCC susceptibility of sensitized Type 304 SS was investigated in SSRT tests. Subsequently, the influence of several carboxylic acids (acetic, formic, lactic, oxalic, propionic, butyric, and valeric) on the SCC behavior of the steel was determined in cyclic loading tests on fracture-mechanics-type specimens in oxygenated water. These organic acids are among those species found in BWR and PWR coolant systems at low concentrations because of ingress and decomposition of organic substances used in the plants. The beneficial effect of some of these substances on SCC of austenitic SSs can be rationalized in terms of the well-known influence of low- 
oxygen concentration on the ECP. 30-35 Although the organic acids could scavenge oxygen, other mechanisms can, in principle, account for their effect on SCC of sensitized Type 304 SS in high-temperature water. 16,36

Fracture-mechanics CGR tests were perlormed on sensitized Type 304 SS in HP water containing $\approx 200 \mathrm{ppb} \mathrm{DO}$. in oxygenated water with 0.1 and $1.0 \mathrm{ppm}$ proplonic or butyric acid, and in water with $1.0 \mathrm{ppm}$ butyric acid and either $100 \mathrm{ppb}$ sulfate or chloride. $38 \mathrm{~A}$ baseline CGR of $2.9 \times 10^{-10} \mathrm{~m} \cdot \mathrm{s}^{-1}$ was obtained in HP water. The addition of $0.1 \mathrm{ppm}$ propionic acid had no effect on the CGR; however, an increase in concentration to $1.0 \mathrm{ppm}$ caused a decrease in the CGR by an order of magnitude. When propionic acid was no longer added to the feedwater, the CGR returned to the initlal baseline value.

When $1.0 \mathrm{ppm}$ butyric acid was added to the oxygenated feedwater, no crack growth was observed over a time interval of $=1150 \mathrm{~h}$ under low-frequency, high-R loading at a $K_{\max }$ value of $=34 \mathrm{MPa} \cdot \mathrm{m}^{1 / 2}$. A decrease in the butyric acid concentration from 1.0 to $0.1 \mathrm{ppm}$ eventually caused the CGR to increase to a value of $1.9 \times 10^{-10} \mathrm{~m} \cdot \mathrm{s}^{-1}$ after $-500 \mathrm{~h}$. The lower concentration of the acid was not sulficlent to inhibit crack growth, as was the case with $0.1 \mathrm{ppm}$ propionic acid in the feedwater.

In axygenated water containing $1 \mathrm{ppm}$ butyric acid and $0.1 \mathrm{ppm}$ sulfate (as $\mathrm{H}_{2} \mathrm{SO}_{4}$ ), the CGR increased significantly to a value of $7.4 \times 10^{-10} \mathrm{~m} \cdot \mathrm{s}^{-1}$. When sulfate was removed from the feedwater, the CGR once agaln decreased to \%ero over a period of $\approx 600 \mathrm{~h}$. Simllarly, when $0.1 \mathrm{ppm}$ chloride (as $\mathrm{NaCl}$ ) was added to the oxygenated feedwater containing $1.0 \mathrm{ppm}$ butyric acid, the CGR of the steel increased to a value of $\approx 1.5 \times 10^{-10} \mathrm{~m} \cdot \mathrm{s}^{-1}$ over an interval of $1150 \mathrm{~h}$. When chloride was not added to the feedwater, the CGR again decreased to zero, even at the relatively high stress intensity factor of $\approx 40 \mathrm{MPa} \cdot \mathrm{m}^{1 / 2}$. The results clearly indicate that the organic acid was not effeclive in inhibiting crack growth in the steel when elther sulfate or chloride was present in the oxygenated water at the $0.1 \mathrm{ppm}$ level.

It is possible that organic acids can inhiblt SCC in oxygenated water even though the ECP regime of the steel is conducive to cracking $\left(>-250 \mathrm{mV}\right.$ [SHE] at $\left.289^{\circ} \mathrm{C}\right)$. Many organic substances such as aliphatic alcohols and acids. carboxylic acids, and others adsorb on metal and oxide electrodes at very high potentlals assoclated with oxygen evolution at amblent temperature. $37-41$ The rate constant for oxygen evolution is independent of the presence of the inhibitor, i.e., the effect of the inhibitor was mainly to block active reaction sites. 37 The adsorbability of the various substances increases as the molecular weight increases, 38.39 and the adsorption follows a logarithmic isotherm at concentrations $210^{-5} \mathrm{M}(\geq 1 \mathrm{ppm}) \cdot 39-41$ It is plausible that carboxylic acids, at a simllar concentration in the water, adsorb on the oxide surface of Type 304 SS and inhibil oxygen reduction, which is the cathodic partial process that couples with anodic dissolution at the crack lip in a slip-dissolution mechanism of crack growth.

The Influence of organic species in normal BWR water on CGRs of recirculation system piping is difficult to quantify. As in the case of chromate when the sulfate levels are very low (<15 ppb), organlc acids (and their precursors that do not contribute to conductivity and are difficult to detect). In facl, may not be deleterious. These specles may, in part, account for the large scatter in the CGR data (1.e.. the low values in the scatter band of CGR data in Figs. 1-5) in HP oxygenated waler. In corrosion-laligue lesting systems, organic substances 
can be present in the feedwater and pass through the water purification system, or be introduced into the feedwater through the cover gas that is typically used to adjust the DO concentration of the water. When this occurs. CGRs can lie in the lower range of the scatter band.

These subtle experimental features can obscure the true effects of DO and impurity concentrations on CGRs and complicate application of laboratory data to predictions of crack growth in piping systems with unique water chemistries that contain corrosion products and impurities at very low concentrations. In some instances, water chemistries (e.g., high chromate/sulfate ratios but with low conductivities, indicative of low concentrations of other ionic species) may produce CGRs below the upper-bound crack-growth curve. Similarly. CGR data obtained in HP oxygenated water containing organic species at low levels can produce low values near the ASME Section XI air line. These data also contribute to the large scatter band.

\section{References}

1. W. J. Shack, in Environmentally Assisted Cracking in Light Water Reactors Semiannual Report, October 1990--March 1991, NUREG/CR-4667 Vol. 12, ANL-91/24, pp. 31-37 (August 1991).

2. J. D. Gilman, R. Rungta, P. Hinds, and H. Mindlan, Corrosion-Fatigue Crack Growth Rates in Austenitic Stainless Steels in Light Water Reaclor Environments. Int. J. Pressure Vessel Piping 31, 55-68 (1988).

3. BWR Hydrogen Water Chemistry Guidelines: 1987 Revision. EPRI NP-4947-SR, Spectal Report, Electric Power Research Institute. Palo Alto, CA (1988).

4. L. A. James and D. P. Jones. Fatigue Crack Growth Correlation for Austenitic Stainless Steels in Air. Proc. Conf. on Predictive Capabilities in Environmentally-Assisted Cracking, R. Rungta, ed.. PVP Vol. 99. American Society of Mechanical Engineers, NY, pp. 363-414 (1985).

5. T. Shoji, H. Takahashi, M. Suzuki, and T. Kondo, A New Parameter for Characterizing Corrosion Fatigue Crack Growth. J. Eng. Mater. Technol. 103, 298-304 (1981).

6. W. E. Ruther, T. F. Kassner, and J. Y. Park, in Environmentally Assisted Cracking in Light Water Reactors Semiannual Report. October 1991--March 1992. NUREG/CR-4667 Vol. 14, ANL-92/30, pp. 33-42 (August 1992).

7. W. E. Ruther, O. K. Chopra, and T. F. Kassner, in Environmentally Assisted Cracking in Light Water Reactors. Semiannual Report, October 1992-March 1993, NUREG/CR-4667 Vol. 16. ANL-93/27, pp. 19-27 (September 1993).

8. L. G. Ljungberg, D. Cubicciolli, and M. Trolle. The Effect of Sulfate on Environmental Cracking in Boiling Waler Reactors under Constant Load or Fatigue, Corrosion 46, 641-652 (1990).

9. L. G. Ljungberg. D. Cubicciotti, and M. Trolle, Effect of Water Impurtites in BWRs on Environmental Crack Growth under Realistic Load Conditions, Proc. 4th Int. Symp. Environmental Degradation of Materials in Nuclear Power Systems-Water Reactors, National Association of Corrosion Engineers, Houston, pp. 4-59 to 4-74 (1990).

10. L. G. Ljungberg, D. Cubicciotll, and M. Trolle. Effect of Water Impurtties in BWRs on Environmental Crack Growth in Type 304 Stainless Steel, NUREG/CP-0112 Vol. 2, ANL-90/22 Vol. 2. pp. 57-64 (August 1990). 
11. J. Alexander et al., Alternative Alloys for BWR Pipe Applications, EPRI NP-2671-LD, pp. 5-17 to 5-32, Electric Power Research Institute, Palo Alto, CA (October 1982).

12. C. W. Jewett and A. E. Pickett, The Benefit of Hydrogen Addition to the Bolling Water Reactor Envtronment on Stress Corrosion Crack Initiation and Growth in Type 304 Stainless Steel, J. Eng. Mater. Technol. 108, 10-19 (1986).

13. P. L. Andresen, Effects of Stress Intensity, $R$ Value, and Environmental Impurities in $288^{\circ} \mathrm{C}$ Water on Crack Growth Rate in Type 304 SS CT Specimens, Corrosion 88, Paper No. 11, St. Louls, MO (March 1988).

14. T. Kawakubo, M. Hishida, K. Amano, and M. Katsuta, Crack Growth Behavior of Type 304 Stainless Steel in Oxygenated $290^{\circ} \mathrm{C}$ Pure Water under Low Frequency Cyclic Loading, Corrosion 36, 638-647 (1980).

15. F. P. Ford, D. F. Taylor, P. L. Andresen, and R. Ballinger, Corrosion-Assisted Cracking of Stainless and Low-alloy Steels, EPRI NP-5064s, Electric Power Research Institute, Palo Alto, CA (February 1987).

16. T. F. Kassner, W. E. Ruther, and W. K. Soppet, Mitigation of Stress Corrosion Cracking of AISI 304 Stainless Steel by Organic Species at Low Concentrations in Oxygenated Water, Corrosion 90, Paper No. 489, Las Vegas, NV (April 1990).

17. W. H. Cullen, R. E. Taylor, K. Törronen, and M. Kemppainen, The Temperature Dependence of Fatigue Crack Growth Rates of A351 CF8A Cast Stainless Steel in LWR Environment, NUREG/CR-3546, MEA-2030 (April 1984).

18. G. Slama, P. Petriquin, S. H. Masson, and T. Mager, Effect of Aging on Mechanical Properties of Austenitic Stainless Steel Castings and Welds, in Light Water Reactor Structural Integrity (Proc. 3rd Int. Seminar on Assuring Structural Integrity of Steel Reactor Pressure Boundary Components), K. E. Stahlkopf and L. E. Steele, eds., Elsevier Applied Science, New York (1984).

19. C. Amzallag, G. Baudry, and J. L. Bernard, Effect of PWR Environment on the Fatigue Crack Growth of Different Stainless Steels and Inconel Type Alloy. Proc. IAEA Specialists' Meeting on Subcritical Crack Growth, NUREG/CP-044 Vol. 1, MEA-2014 Vol. 1 (May 1983).

20. W. H. Bamford, Fatigue Crack Growth of Stainless Steel Piping in a Pressurized Water Reactor Environment, J. Pressure Vessel Technol. 101, 73-79 (1979).

21. J. L. Bernard and G. S. Slama, Fatigue Crack Growth Curve in Air Envtronment at $300^{\circ} \mathrm{C}$ for Stainless Steels, Nucl. Technol. 68, 136-146 (1982).

22. W. E. Ruther, W. K. Soppet, and T. F. Kassner, in Environmentally Assisted Cracking in Light Water Reactors, Annual Report, October 1983-September 1984, NUREG/CR4287, ANI-85-33, pp. 93-1 13 (June 1985).

23. F. P. Ford, A Mechanism of Environmentally-Controlled Crack Growth of Structural Steels in High-Temperature Water, Proc. IAEA Specialists' Meeting on Subcritical Crack Growth, NUREG/CP-044 Vol. 2, MEA-2014 Vol. 2 (May 1983).

24. W. E. Ruther, W. J. Shack, T. F. Kassner, and W. K. Soppet, in Environmentally Assisted Cracking in Light Water Reactors: Semiannual Report, Aprtl 1988 - September 1988 , NUREG/CR-4667 Vol. 7, ANL-89/40, pp. 3-14 (March 1990).

25. P. L. Andresen, Effects of Temperature on Crack Growth Rate in Sensitized Type 304 Stainless Steel and Alloy 600, Corrosion 49, 714-725 (1993).

26. W. E. Ruther, W. K. Soppet, and T. F. Kassner, in Environmentally Assisted Cracking in Light Water Reactors: Semiannual Report, April - September 1991, NUREG/CR-4667 Vol. 13, ANL-92/6, pp. 16-19 (March 1992). 
27. B. H. Dillman, R. A. Reed, and C. C. Lin, BWR Coolant Impurtity Identification Study, Final Report, EPRI NP-4156 (August 1985).

28. B. H. Dillman, J. C. Elliot, R. A. Head, J. E. Osterle, and R. S. Tunder, Monitoring of Chemical Contaminants in BWRs, Final Report, EPRI NP-4134 (July 1985).

29. J. E. Richards and W. A. Byers, Industry-wide Survey of PWR Organics, Final Report, EPRI NP-4698 (July 1986).

30. W. E. Ruther, W. K. Soppet, and T. F. Kassner, in Environmentally Assisted Cracking in Light Water Reactors: Semiannual Report, April - September 1987, NUREG/CR-4667 Vol. V, ANL-88-32 (February 1989), pp. 24-37.

31. W. E. Ruther, W. K. Soppet, and T. F. Kassner, in Environmentally Assisted Cracking in Light Water Reactors: Semiannual Report, October 1987 - March 1988, NUREG/CR4667 Vol. 6, ANL-89/10 (August 1989), pp. 27-42.

32. W. E. Ruther, W. K. Soppet, and T. F. Kassner, in Light-Water-Reactor Safety Research Programs: Quarterly Progress Report, January - March 1985, NUREG/CR-4490 Vol. I, ANL-85-75 Vol. I (March 1986), pp. 25-42.

33. J. N. Kass and R. L. Cowan, Hydrogen Water Chemistry for BWRs, in Environmental Degradation of Materials in Nuclear Power Systems-Water Reactors, Proc. of the 2nd Int. Symp., Monterey, CA, ANS, La Grange Park, IL (1986), pp. 211-218.

34. M. E. Indig, B. M. Gordon, R. B. Davis, and J. E. Weber, Evaluation of In-reactor Stress Corrosion Cracking via Electrochemical Measurements, Ibid., pp. 411-418.

35. D. D. Macdonald and G. Cragnolino, The Critical Potential for IGSCC of Sensitized Type 304 SS in High Temperature Aqueous Systems, ibid., pp. 426-434.

36. W. E. Ruther, W. K. Soppet, and T. F. Kassner, in Environmentally Assisted Cracking in Light Water Reactors: Semiannual Report, April 1988 - September 1988, NUREG/CR4667 Vol. 7, ANL-89/40, pp. 29-37 (March 1990).

37. Yu. M. Tyurin, G. F. Volodin, and V. S. Paducheva, Elektrokhimiya 7, 1309-1312 (1971).

38. V. I. Naumov, L. A. Smirnova, G. N. Afon'shin, and Yu. M. Tyurin, Elektrokhimiya 7. 1872-1876 (1971).

39. Yu. M. Tyurin, V. I. Naumov, Vyach. N. Flerov, and Yu. V. Battalova, Elektrokhimiya 10. 1119-1123 (1974).

40. V. I. Naumov and Yu. M. Tyurin, Elektrokhimiya 8, 1220-1223 (1972).

41. Yu. M. Tyurin, G. F. Volodin, L. A. Smirnova, and Yu. V. Battalova, Elektrokhimiya 9 , 532-536 (1973).

42. Proceedings of the EPRI Workshop on Significance and Control of Chromate in BWRs, Chicago, IL, November 13-14, 1990.

43-48. Not cited.

49. W. E. Ruther, W. K. Soppet, and T. F. Kassner, in Materials Science and Technology Division Light-Water-Reactor Safety Research Program: Quarterly Progress Report, October - December 1983, NUREG/CR-3689 Vol. IV, ANL-83-85 Vol. IV, pp. 75-87 (August 1984).

50. W. E. Ruther, W. K. Soppet, and T. F. Kassner, in Environmentally Assisted Cracking in Light Water Reactors: Annual Report, October 1983 - September 1984, NUREG/CR4287, ANL-85-33, pp. 110-113 (June 1985). 
51. W. E. Ruther, W. K. Soppet, J. Y. Park, and T. F. Kassner, in Environmentally Asststed Cracking in Light Water Reactors: Semiannual Report, April - September 1986. NUREG/CR-4667 Vol. III, ANL-87-37, pp. 2-7 (September 1987).

52. W. E. Ruther, W. K. Soppet, J. Y. Park, and T. F. Kassner, in Environmentally Assisted Cracking in Light Water Reactors: Semiannual Report, October 1986 - March 1987. NUREG/CR-4667 Vol. IV, ANL-87-41, pp. 2-10 (December 1987).

53. W. E. Ruther, W. K. Soppet, J. Y. Park, and T. F. Kassner, In Envtronmentally Assisted Cracking in Light Water Reactors: Semiannual Report, Aprll - September 1987. NUREG/CR-4667 Vol. V, ANL-88-32, pp. 3-9 (February 1989).

54. W. E. Ruther, W. K. Soppet, J. Y. Park, and T. F. Kassner, in Environmentally Assisted Cracking in Light Water Reactors: Semiannual Report, October 1987 - March 1988, NUREG/CR-4667 Vol. VI, ANL-89/10, pp. 5-14 (August 1989).

55. W. E. Ruther, W. J. Shack, T. F. Kassner, and W. K. Soppet, in Environmentally Assisted Cracking in Light Water Reactors: Semiannual Report, Aprll 1988 - September 1988 , NUREG/CR-4667 Vol. 7, ANL-89/40, pp. 3-14 (March 1990).

56. W. E. Ruther, J. Y. Park, T. F. Kassner, and W. K. Soppet, in Environmentally Assisted Cracking in Light Water Reactors: Semiannual Report, October 1988 - March 1989. NUREG/CR-4667 Vol. 8, ANL-90/4, pp. 2-15 (June 1990).

57. W. E. Ruther, W. K. Soppet, and T. F. Kassner, in Environmentally Assisted Cracking in Light Water Reactors: Semiannual Report, October 1990 - March 1991. NUREG/CR-4667 Vol. 12, ANL-91/24, pp. 22-30 (August 1991).

58. W. E. Ruther, W. K. Soppet, and T. F. Kassner, in Environmentally Assisted Cracking in Light Water Reactors: Semiannual Report, April - September 1991. NUREG/CR-4667 Vol. 13, ANL-92/6, pp. 16-19 (March 1992).

59. J. Y. Park and W. J. Shack, in Light-Water-Reactor Safety Research Programs: Quarterly Progress Report, October - December 1984, NUREG/CR-3998 Vol. III, ANL-84-60 Vol. III, pp. 7-14 (October 1985).

60. J. Y. Park and W. J. Shack, in Light-Water-Reactor Safety Research Programs: Quarterly Progress Report, January - March 1985, NUREG/CR-4490 Vol. I, ANL-85-75 Vol. I, pp. 14-16 and 43-49 (March 1986).

61. W. E. Ruther, W. K. Soppet, J. Y. Park, and T. F. Kassner, in Materials Sclence and Technology Division Light-Water-Reactor Safety Research Program: Quarterly Proyress Report, April - June 1983, NUREG/CR-3689 Vol. II, ANL-83-85 Vol. II, pp. 44-48 (June 1984).

62. J. Y. Park and W. J. Shack, in Environmentally Assisted Cracking in Light Water Reactors: Semiannual Report, April 1989 - September 1989, NUREG/CR-4667 Vol. 9, ANL-90/48, pp. 3-6 (March 1991). 


\section{Distribution for NUREG/CR-6176 (ANL-94/1)}

Internal

W. J. Shack (45)

TIS Files

\section{External}

NRC, for distribution per R5

Libraries

ANL-E

ANL-W

Manager, Chicago Field Office, DOE

Energy Technology Division Review Committee:

H. K. Birnbaum, University of Illinois, Urbana

R. C. Buchanan, University of Cincinnati, Cincinnati

M. S. Dresselhaus, Massachusetts Institute of Technology, Cambridge, MA

B. G. Jones, University of Illinois, Urbana

C.-Y. Li, Cornell University, Ithaca, NY

S. N. Liu, Fremont, CA

R. E. Smith, SciTech, Inc., Morrisville, NC

P. L. Andresen, General Electric Corporate Research and Development, Schenectady, NY

R. G. Ballinger, Massachusetts Institute of Technology, Cambridge, MA

W. H. Bamford, Westinghouse Nuclear Energy Systems, Pittsburgh

K. K. Bandyopadhyay, Brookhaven National Laboratory

S. M. Bruemmer, Battelle Pacific Northwest Laboratory

J. A. Christensen, Battelle Pacific Northwest Laboratory

H. S. Chung, Korea Atomic Energy Research Institute, Daejeon, Korea

G. Cragnolino, Southwest Research Inst., San Antonio, TX

W. H. Cullen, Materials Engineering \& Assoc., Inc., Lanham, MD

A. F. Deardorff, Structural Integrity Associates, Inc., San Jose, CA

R. Duncan, ABB CE Nuclear Power, Windsor, CT

E. D. Eason, Modeling and Computing Services, Newark, CA

M. Fox, APTECH, Tucson, AZ

D. G. Franklin, Bettis Atomic Power Laboratory

Y. S. Garud, S. Levy, Inc., Campbell, CA

F. Garzarolli, KWU, Erlangen, Germany

J. D. Gilman, Electric Power Research Inst., Palo Alto, CA

B. M. Gordon, General Electric Co., San Jose, CA

J. W. Halley, U. Minnesota, Minneapolis 
H. E. Hanninen, Technical Research Centre of Finland, Espoo

D. Harrison, USDOE, Germantown, MD

J. Hickling, CML Capcis March Ltd., Erlangen-Tennonlohe, Germany

C. L. Hoffmann, ABB CE Nuclear Power, Windsor, CT

M. E. Indig, General Electric Co., Pleasanton, CA

H. S. Isaaci, Brookhaven National Laboratory

A. Jacobs, General Electric Co., San Jose, CA

L. James, Bettis Atomic Power Laboratory

C. Jansson, Vattenfall Energisystem, Vallingby, Sweden

D. F. Jones, Bettis Atomic Power Laboratory, West Mifflin, PA

R. H. Jones, Battelle Pactfic Northwest Laboratory

R. L. Jones, Electric Power Research Institute, Palo Alto, CA

T. Karlsen, OECD Halden Reactor Project, Halden, Norway

C. Kim, Westinghouse Electric Corp., Pittsburgh

W. C. Kroenke, Bettis Atomic Power Laboratory

L. LJungberg, ASEA-ATOM, Vasteras, Sweden

C. D. Lundin, U. Tennessee, Knoxville

D. D. Macdonald, Pennsylvania State University, University Park

T. R. Mager, Westinghouse Electric Corp., Pittsburgh

R. D. McCright, Lawrence Livermore National Laboratory

H. Metha, General Electric Co., San Jose, CA

D. Morgan, Pennsylvania Power and Light Co., Allentown, PA

J. L. Nelson, Electric Power Research Inst., Palo Alto, CA

W. J. O'Donnell, O'Donnell \& Associates, Inc., Pittsburgh

M. Prager, Pressure Vessel Research Council, New York, NY

S. Ranganath, General Electric Co., San Jose, CA

P. M. Scott, Framatome, Paris, France

C. Shepherd, AEA Technology-Harwell Labs., Didcot, Oxon, UK

S. Smialowska, Ohio State University, Columbus

M. O. Speidel, Swiss Federal Institute of Technology, Zurich, Switzerland

D. M. Stevens, Lynchburg Research Center, Babcock \& Wilcox Co., Lynchburg, VA

W. A. Van Der Sluys, Research \& Development Division, Babcock \& Wilcox Co., Alliance, $\mathrm{OH}$

J. C. Van Duysen, Electricite de France-Research and Development Centre de Renardieres, Moret-Sur-Loing, France

E. Venerus, Knolls Atomic Power Laboratory

J. R. Weeks, Brookhaven National Laboratory

D. Winkel, Teleco Oil Field Services, Meriden, CT

S. Yukawa, Boulder, CO 

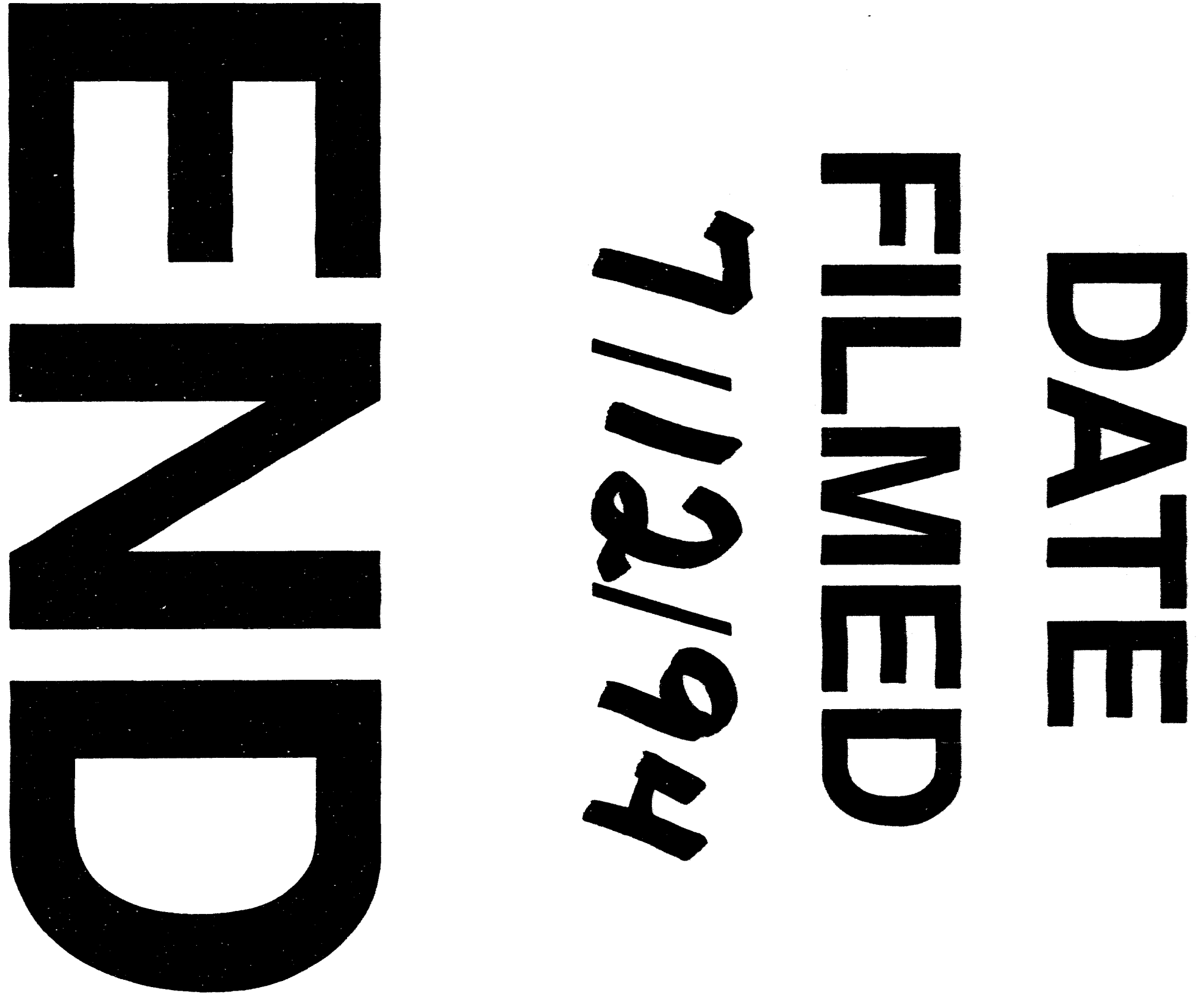

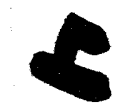


(r)

- - 